\title{
Interval-Valued Hesitant Fuzzy Hamacher Synergetic Weighted Aggregation Operators and Their Application to Shale Gas Areas Selection
}

\author{
Liang-Guo $\mathrm{Li}^{1}$ and Ding-Hong Peng ${ }^{1,2}$ \\ ${ }^{1}$ Kunming University of Science and Technology, Kunming 650093, China \\ ${ }^{2}$ School of Management, Harbin University of Science and Technology, Harbin 150040, China \\ Correspondence should be addressed to Ding-Hong Peng; pengdinghong2009@163.com
}

Received 26 October 2013; Revised 21 January 2014; Accepted 23 January 2014; Published 27 April 2014

Academic Editor: Wudhichai Assawinchaichote

Copyright (c) 2014 L.-G. Li and D.-H. Peng. This is an open access article distributed under the Creative Commons Attribution License, which permits unrestricted use, distribution, and reproduction in any medium, provided the original work is properly cited.

\begin{abstract}
We investigate the multiple criteria decision making (MCDM) problem concerns on the selection of shale gas areas with intervalvalued hesitant fuzzy information. First, some Hamacher operations of interval-valued hesitant fuzzy information are introduced, which generalize and extend the existing ones. Then some interval-valued hesitant fuzzy Hamacher weighted aggregation operators, especially, the interval-valued hesitant fuzzy Hamacher synergetic weighted averaging (IVHFHSWA) operators and their geometric version (IVHFHSWG) operators that weight simultaneously the argument variables themselves and their position orders and thus generalize the ideas of the weighted averaging and the ordered weighted averaging, are proposed. The distinct advantages of these operators are that they can provide more choices for the decision makers and considerably enhance or deteriorate the performance of aggregation. The essential properties of these operators are studied and their specific cases are discussed. Based on the IVHFHSWA operator, we propose a practical approach to shale gas areas selection with interval-valued hesitant fuzzy information. Finally, an illustrative example for selecting the shale gas areas is used to demonstrate the practicality and effectiveness of the proposed approach and a comparative analysis is performed with other approaches to highlight the distinctive advantages of the proposed operators.
\end{abstract}

\section{Instruction}

As a novel generalization of fuzzy sets, hesitant fuzzy sets (HFSs) [1,2] introduced by Torra and Narukawa have been successfully used in the decision making field as a powerful tool for processing with uncertain and vague information. Unlike the other generalizations of fuzzy sets, HFSs, which permit the membership degree of an element to a set to be represented as several possible values between 0 and 1 , are quite suited for describing the situation where we have a set of possible values, rather than a margin of error or some possibility distribution on the possible values, and thus HFSs are very useful in dealing with the practical decision making situations where people hesitate among several values to express their opinions [3-5] or their opinions with incongruity [6-8], especially, the group decision making with anonymity [9-12]. Moreover, HFSs could also avoid performing information aggregation and can directly reflect the differences of the opinions of different experts $[1,13,14]$. In addition, it is proven that the envelope of hesitant fuzzy set is an intuitionistic fuzzy set (IFS); all HFSs are type-2 fuzzy set and hesitant fuzzy set and fuzzy multiset have the same form, but their operations are different [2]. Thus, HFSs open new perfectives for further research on decision making under hesitant environments and have received much attention from many authors. Torra and Narukawa $[1,2]$ proposed some set theoretic operations such as union, intersection, and complement on HFSs. Subsequently, Xia and Xu [6] defined some new operations on HFSs based on the interconnection between HFSs and the IFSs and then made an intensive study of hesitant fuzzy information aggregation techniques and their applications in decision making. Xu and Xia [7] 
investigated some distance measures for HFSs drawing on the well-known Hamming distance, the Euclidean distance, the Hausdorff metric, and their generalizations. Following these pioneering studies, many subsequent studies on the aggregation operators $[8,9,12-15]$, the discrimination measures [16] (including distance measures [3-5, 13-15], similarity measures $[3,7]$, correlation measures $[3,17]$, entropy, and cross-entropy [18]) for hesitant fuzzy sets (HFSs), and the further extensions of the HFSs, such as the interval-values HFSs (IVHFSs) [11, 17], the dual (or generalized) HFSs (DHFSs) $[10,19]$, and the hesitant fuzzy linguistic term sets (HFLTSs) [20, 21], have been conducted.

In some practical decision making problems, however, the precise membership degrees of an element to a set are sometimes hard to be specified. To overcome the barrier, Chen et al. [11, 17] proposed the concept of interval-valued hesitant fuzzy sets (IVHFSs) that represent the membership degrees of an element to a set with several possible interval values and then presented some interval-valued hesitant fuzzy aggregation operators. Wei [22] developed some hesitant interval-valued fuzzy aggregation operators (which are essential interval-valued hesitant fuzzy aggregation operators), such as the hesitant interval-valued fuzzy weighted aggregation operators (HIVFWA and HIVFWG), the hesitant interval-valued fuzzy ordered weighted aggregation operators (HIVFOWA and HIVFOWG), the hesitant intervalvalued fuzzy choquet ordered aggregation operators (HIVFCOA and HIVFCOG), the hesitant interval-valued fuzzy prioritized aggregation operator and the hesitant intervalvalued fuzzy power aggregation operator.

It is well known that the aggregation operators introduced above are based on the basic algebraic $t$-norms ( $t$-norm and $t$-conorm) of HFSs (or IVHFSs) for carrying the combination process, which are not the unique $t$-norms that can be chosen to model the intersection and union of HFSs (or IVHFSs). For instance, Wei and Zhao [23] presented the Einstein operations of interval-valued hesitant fuzzy sets based the Einstein $t$-norms and then developed some interval-valued hesitant fuzzy Einstein aggregation operators and induced intervalvalued hesitant fuzzy Einstein aggregation operators. Besides, there are a lot of $t$-norms that can be used to construct the operations of HFSs (or IVHFSs); one of them is the Hamacher $t$-norms [24-27], which have proven that the basic algebraic $t$-norms and the Einstein $t$-norms are the special cases of the Hamacher $t$-norms and can supply a wide class of $t$ norm operators ranging from the probabilistic product to the weakest $t$-norm by the choice of a parameter. Thus the Hamacher $t$-norms can considerably enhance or deteriorate the performance of aggregation. Given the advantages of the Hamacher $t$-norms, in this paper, we will investigate the interval-valued hesitant fuzzy aggregation operators based on the Hamacher $t$-norms and apply them to the multiple criteria decision making.

To do so, the remainder of this paper is organized as follows. Section 2 introduces some preliminary concepts related to the interval-valued hesitant fuzzy sets and their operations based on the Hamacher $t$-norms. In Section 3, based on the defined operations, we first develop the interval-valued hesitant fuzzy Hamacher weighted averaging operators and the interval-valued hesitant fuzzy Hamacher ordered weighted averaging operators, then, based on which, we further propose the interval-valued hesitant fuzzy Hamacher synergetic weighted aggregation operators that simultaneously consider the weights of argument variables themselves and their position orders. Moreover, some essential properties and special cases of these operators are studied. In Section 4, we develop a practical approach based on the IVHFHSWA operators to multicriteria decision making under interval-valued hesitant fuzzy environments. Section 5 an illustrative example for selecting the shale gas areas is used to demonstrate the practicality and effectiveness of the proposed approach and Section 6 a comparative analysis is performed with other approaches to highlight the distinctive advantages of the proposed operators. Finally, we summarize the main conclusions of the paper in Section 7.

\section{Preliminaries}

To overcome the barrier that the precise membership degrees of an element to a set are sometimes hard to be specified, Chen et al. [11, 17] introduce interval-valued hesitant fuzzy set (IVHFS), that represents the membership degrees of an element to a set with several possible interval values.

Definition 1 (see [11]). Let $X$ be a reference set and let $D[0,1]$ be the set of all closed subintervals of $[0,1]$. Then an IVHFS on $X$ is defined as

$$
\widetilde{E}=\left\{\left\langle x, \widetilde{h}_{\widetilde{E}}(x)\right\rangle \mid x \in X\right\},
$$

where $\widetilde{h}_{\widetilde{E}}(x): x \rightarrow D[0,1]$ denotes all possible intervalvalued membership degrees of the element $x \in X$ to the set $\widetilde{E}$. For convenience, we call $\widetilde{h}_{\widetilde{E}}(x)$ an interval-valued hesitant fuzzy element (IVHFE), which reads

$$
\widetilde{h}_{\widetilde{E}}(x)=\bigcup_{\tilde{\gamma} \in \widetilde{h}_{\tilde{E}}(x)}\left\{\tilde{\gamma}=\left[\tilde{\gamma}^{L}, \widetilde{\gamma}^{U}\right] \mid 0 \leq \widetilde{\gamma}^{L} \leq \widetilde{\gamma}^{U} \leq 1\right\} .
$$

The operational laws of IVHFSs can be constructed by $t$-norms ( $t$-norm and $t$-conorm), which satisfy the requirements of the conjunction and disjunction operators, respectively. The existing interval-valued hesitant fuzzy operational laws include the ones based on the algebraic $t$-norms [6, $11,13-15,22]$ and the ones based on the Einstein $t$-norms [23]. It is well known that the Hamacher $t$-norms are more generalized and flexible than the algebraic $t$-norms and the Einstein $t$-norms, and they are defined as follows.

Definition 2 (see $[24,25])$. The Hamacher $t$-norm $\phi_{\eta}$ and its conorm $\varphi_{\eta}$ are defined as

$$
\begin{array}{ll}
\phi_{\eta}(x, y)=\frac{x y}{\eta+(1-\eta)(x+y-x y)}, & \eta>0, \\
\varphi_{\eta}(x, y)=\frac{x+y-x y-(1-\eta) x y}{1-(1-\eta) x y}, & \eta>0 .
\end{array}
$$

Similar to the existing operations of IVHFEs, based on the Hamacher $t$-norms, we can establish some fundamental Hamacher operations of IVHFEs. 
Definition 3. Let $\widetilde{h}, \widetilde{h}_{1}$, and $\tilde{h}_{2}$ be three IVHFEs; then the Hamacher operations of IVHFSs are defined as follows:

(1)

$$
\begin{array}{r}
\lambda \widetilde{h}=\bigcup_{\tilde{\gamma} \in \tilde{h}}\left\{\left[\frac{\left(1+(\eta-1) \tilde{\gamma}^{L}\right)^{\lambda}-\left(1-\tilde{\gamma}^{L}\right)^{\lambda}}{\left(1+(\eta-1) \tilde{\gamma}^{L}\right)^{\lambda}+(\eta-1)\left(1-\tilde{\gamma}^{L}\right)^{\lambda}},\right.\right. \\
\\
\left.\left.\quad \frac{\left(1+(\eta-1) \tilde{\gamma}^{U}\right)^{\lambda}-\left(1-\tilde{\gamma}^{U}\right)^{\lambda}}{\left(1+(\eta-1) \tilde{\gamma}^{U}\right)^{\lambda}+(\eta-1)\left(1-\tilde{\gamma}^{U}\right)^{\lambda}}\right]\right\},
\end{array}
$$$$
(\lambda>0)
$$

(2)

$$
\begin{aligned}
\tilde{h}^{\lambda}=\bigcup_{\tilde{\gamma} \in \tilde{h}}\left\{\left[\frac{\eta\left(\tilde{\gamma}^{L}\right)^{\lambda}}{\left(1+(\eta-1)\left(1-\tilde{\gamma}^{L}\right)\right)^{\lambda}+(\eta-1)\left(\tilde{\gamma}^{L}\right)^{\lambda}},\right.\right. \\
\\
\left.\left.\frac{\eta\left(\tilde{\gamma}^{U}\right)^{\lambda}}{\left(1+(\eta-1)\left(1-\tilde{\gamma}^{U}\right)\right)^{\lambda}+(\eta-1)\left(\tilde{\gamma}^{U}\right)^{\lambda}}\right]\right\}, \\
(\lambda>0) ;
\end{aligned}
$$

(3)

$$
\begin{aligned}
& \tilde{h}_{1} \oplus \tilde{h}_{2} \\
& =\bigcup_{\widetilde{\gamma}_{1} \in \widetilde{h}_{1}, \widetilde{\gamma}_{2} \in \widetilde{h}_{2}}\left\{\left[\frac{\tilde{\gamma}_{1}^{L}+\tilde{\gamma}_{2}^{L}-\tilde{\gamma}_{1}^{L} \widetilde{\gamma}_{2}^{L}-(1-\eta) \tilde{\gamma}_{1}^{L} \widetilde{\gamma}_{2}^{L}}{1-(1-\eta) \tilde{\gamma}_{1}^{L} \widetilde{\gamma}_{2}^{L}},\right.\right. \\
& \left.\left.\frac{\tilde{\gamma}_{1}^{U}+\tilde{\gamma}_{2}^{U}-\tilde{\gamma}_{1}^{U} \tilde{\gamma}_{2}^{U}-(1-\eta) \tilde{\gamma}_{1}^{U} \tilde{\gamma}_{2}^{U}}{1-(1-\eta) \tilde{\gamma}_{1}^{U} \tilde{\gamma}_{2}^{U}}\right]\right\}
\end{aligned}
$$

$$
\begin{aligned}
\tilde{h}_{1} \otimes \tilde{h}_{2} & \\
= & \bigcup_{\tilde{\gamma}_{1} \in \tilde{h}_{1}, \widetilde{\gamma}_{2} \in \tilde{h}_{2}}\left\{\left[\frac{\tilde{\gamma}_{1}^{L} \tilde{\gamma}_{2}^{L}}{\eta-(1-\eta)\left(\tilde{\gamma}_{1}^{L}+\tilde{\gamma}_{2}^{L}-\tilde{\gamma}_{1}^{L} \tilde{\gamma}_{2}^{L}\right)},\right.\right. \\
& \left.\left.\frac{\tilde{\gamma}_{1}^{U} \tilde{\gamma}_{2}^{U}}{\eta-(1-\eta)\left(\tilde{\gamma}_{1}^{U}+\tilde{\gamma}_{2}^{U}-\tilde{\gamma}_{1}^{U} \tilde{\gamma}_{2}^{U}\right)}\right]\right\} .
\end{aligned}
$$

The Hamacher operations of IVHFEs contain a wide class of special cases. Especially, if $\eta=1$, then we have (1)-(4) reduced to the following forms, which are presented by Chen et al. [11]:

$\left(1^{\prime}\right)$

$$
\lambda \widetilde{h}=\bigcup_{\tilde{\gamma} \in \tilde{h}}\left\{\left[1-\left(1-\tilde{\gamma}^{L}\right)^{\lambda}, 1-\left(1-\widetilde{\gamma}^{U}\right)^{\lambda}\right]\right\} ;
$$

$\left(2^{\prime}\right)$

$$
\tilde{h}^{\lambda}=\bigcup_{\tilde{\gamma} \in \tilde{h}}\left\{\left[\left(\tilde{\gamma}^{L}\right)^{\lambda},\left(\widetilde{\gamma}^{U}\right)^{\lambda}\right]\right\}
$$

$$
\begin{array}{r}
\tilde{h}_{1} \oplus \tilde{h}_{2}=\bigcup_{\tilde{\gamma}_{1} \in \tilde{h}_{1}, \tilde{\gamma}_{2} \in \tilde{h}_{2}}\left\{\left[\tilde{\gamma}_{1}^{L}+\tilde{\gamma}_{2}^{L}-\tilde{\gamma}_{1}^{L} \tilde{\gamma}_{2}^{L},\right.\right. \\
\left.\left.\widetilde{\gamma}_{1}^{U}+\widetilde{\gamma}_{2}^{U}-\widetilde{\gamma}_{1}^{U} \widetilde{\gamma}_{2}^{U}\right]\right\} ;
\end{array}
$$

$$
\tilde{h}_{1} \otimes \tilde{h}_{2}=\bigcup_{\tilde{\gamma}_{1} \in \tilde{h}_{1}, \tilde{\gamma}_{2} \in \tilde{h}_{2}}\left\{\left[\tilde{\gamma}_{1}^{L} \tilde{\gamma}_{2}^{L}, \widetilde{\gamma}_{1}^{U} \widetilde{\gamma}_{2}^{U}\right]\right\} .
$$

If $\eta=2$, then we have (1)-(4) reduced to the following forms, which are presented by Wei and Zhao [23]:

$\left(1^{\prime \prime}\right)$

$$
\begin{aligned}
\lambda \tilde{h}=\bigcup_{\tilde{\gamma} \in \tilde{h}}\{ & {\left[\frac{\left(1+\tilde{\gamma}^{L}\right)^{\lambda}-\left(1-\tilde{\gamma}^{L}\right)^{\lambda}}{\left(1+\tilde{\gamma}^{L}\right)^{\lambda}+\left(1-\tilde{\gamma}^{L}\right)^{\lambda}},\right.} \\
& \left.\left.\frac{\left(1+\widetilde{\gamma}^{U}\right)^{\lambda}-\left(1-\widetilde{\gamma}^{U}\right)^{\lambda}}{\left(1+\tilde{\gamma}^{U}\right)^{\lambda}+\left(1-\tilde{\gamma}^{U}\right)^{\lambda}}\right]\right\} ;
\end{aligned}
$$

$\left(2^{\prime \prime}\right)$

$$
\tilde{h}^{\lambda}=\bigcup_{\tilde{\gamma} \in \tilde{h}}\left\{\left[\frac{2\left(\tilde{\gamma}^{L}\right)^{\lambda}}{\left(2-\tilde{\gamma}^{L}\right)^{\lambda}+\left(\tilde{\gamma}^{L}\right)^{\lambda}}, \frac{2\left(\tilde{\gamma}^{U}\right)^{\lambda}}{\left(2-\tilde{\gamma}^{U}\right)^{\lambda}+\left(\tilde{\gamma}^{U}\right)^{\lambda}}\right]\right\} ;
$$

$\left(3^{\prime \prime}\right)$

$$
\tilde{h}_{1} \oplus \tilde{h}_{2}=\bigcup_{\tilde{\gamma}_{1} \in \tilde{h}_{1}, \widetilde{\gamma}_{2} \in \tilde{h}_{2}}\left\{\left[\frac{\tilde{\gamma}_{1}^{L}+\tilde{\gamma}_{2}^{L}}{1+\tilde{\gamma}_{1}^{L} \widetilde{\gamma}_{2}^{L}}, \frac{\tilde{\gamma}_{1}^{U}+\widetilde{\gamma}_{2}^{U}}{1+\tilde{\gamma}_{1}^{U} \widetilde{\gamma}_{2}^{U}}\right]\right\}
$$

$\left(4^{\prime \prime}\right)$

$\tilde{h}_{1} \otimes \tilde{h}_{2}=\bigcup_{\tilde{\gamma}_{1} \in \widetilde{h}_{1}, \tilde{\gamma}_{2} \in \tilde{h}_{2}}\left\{\left[\frac{\tilde{\gamma}_{1}^{L} \tilde{\gamma}_{2}^{L}}{1+\left(1-\tilde{\gamma}_{1}^{L}\right)\left(1-\tilde{\gamma}_{2}^{L}\right)}\right.\right.$,

$$
\left.\left.\frac{\widetilde{\gamma}_{1}^{U} \widetilde{\gamma}_{2}^{U}}{1+\left(1-\widetilde{\gamma}_{1}^{U}\right)\left(1-\widetilde{\gamma}_{2}^{U}\right)}\right]\right\}
$$


Based on Definition 3, the following Theorem 4 can be easily proven.

Theorem 4. Let $\widetilde{h}_{1}$ and $\widetilde{h}_{2}$ be two IVHFEs; then

(1) $\tilde{h}_{1} \oplus \tilde{h}_{2}=\tilde{h}_{2} \oplus \tilde{h}_{1}$,

(2) $\widetilde{h}_{1} \otimes \widetilde{h}_{2}=\widetilde{h}_{2} \otimes \widetilde{h}_{1}$,

(3) $\lambda_{1} \widetilde{h}_{1} \oplus \lambda_{2} \widetilde{h}_{1}=\left(\lambda_{1}+\lambda_{2}\right) \widetilde{h}_{1}, \lambda_{1}, \lambda_{2}>0$,

(4) $\lambda\left(\widetilde{h}_{1} \oplus \widetilde{h}_{2}\right)=\lambda \widetilde{h}_{2} \oplus \lambda \widetilde{h}_{1}, \lambda>0$,

(5) $\tilde{h}_{1}^{\lambda_{1}} \otimes \tilde{h}_{1}^{\lambda_{2}}=\tilde{h}_{1}^{\left(\lambda_{1}+\lambda_{2}\right)}, \lambda_{1}, \lambda_{2}>0$,

(6) $\tilde{h}_{2}^{\lambda} \otimes \widetilde{h}_{1}^{\lambda}=\left(\widetilde{h}_{1} \otimes \widetilde{h}_{2}\right)^{\lambda}, \lambda>0$.

The proofs of Theorem 4 are straightforward and omitted here for saving space.

Chen et al. [11] defined the score function of IVHFE, and gave a comparison approach of the score values of two IVHFEs with the possibility degree.

Definition 5. For an IVHFE $\tilde{h}, s(\widetilde{h})=(1 / \# \widetilde{h}) \sum_{\tilde{\gamma} \in \tilde{h}} \tilde{\gamma}=$ $\left[(1 / \# \widetilde{h}) \sum_{\tilde{\gamma} \in \tilde{h}} \tilde{\gamma}^{L},(1 / \# \tilde{h}) \sum_{\tilde{\gamma} \in \tilde{h}} \widetilde{\gamma}^{U}\right]$ is called the score function of $\widetilde{h}$. Moreover, for two IVHFEs, $h_{1}$ and $h_{2}$, if

$$
\begin{gathered}
P\left(\widetilde{h}_{1} \geq \widetilde{h}_{2}\right) \\
=\max \left\{1-\max \left(\left(\frac{1}{\# \widetilde{h}_{2}} \sum_{\tilde{\gamma}_{2} \in \widetilde{h}_{2}} \widetilde{\gamma}_{2}^{U}-\frac{1}{\# \widetilde{h}_{1}} \sum_{\widetilde{\gamma}_{1} \in \widetilde{h}_{1}} \widetilde{\gamma}_{1}^{L}\right)\right.\right. \\
\times\left(\frac{1}{\# \widetilde{h}_{1}} \sum_{\widetilde{\gamma}_{1} \in \widetilde{h}_{1}}\left(\widetilde{\gamma}_{1}^{U}-\widetilde{\gamma}_{1}^{L}\right)\right. \\
\left.+\frac{1}{\# \widetilde{h}_{2}} \sum_{\tilde{\gamma}_{2} \in \widetilde{h}_{2}}\left(\widetilde{\gamma}_{2}^{U}-\widetilde{\gamma}_{2}^{L}\right)\right)^{-1}, \\
0), 0\}>0.5,
\end{gathered}
$$

then $\tilde{h}_{1}>\widetilde{h}_{2}$; if $P\left(\widetilde{h}_{1} \geq \widetilde{h}_{2}\right)=0.5$, then $\tilde{h}_{1}=\widetilde{h}_{2}$.

Based on the possibility degree, we further give the definition of the relative possibility degree to rank or compare multiple IVHFEs $\widetilde{h}_{j}(j=1,2, \ldots, n)$.
Definition 6. Let $\widetilde{h}_{j}=\bigcup_{\tilde{\gamma}_{j} \in \tilde{h}_{j}}\left\{\tilde{\gamma}_{j}=\left[\tilde{\gamma}_{j}^{L}, \widetilde{\gamma}_{j}^{U}\right]\right\}(j=1,2, \ldots, n)$ be a collection of IVHFEs, then the relative possibility degree of $\widetilde{h}_{j}$ that dominates all $\widetilde{h}_{k}(k=1,2, \ldots, n)$ is defined as

$$
\begin{aligned}
& P\left(\tilde{h}_{j}\right)=\frac{1}{n(n-1)} \\
& \times\left(\sum _ { k = 1 } ^ { n } \operatorname { m a x } \left\{1-\max \left(\left(\frac{1}{\# \widetilde{h}_{k}} \sum_{\widetilde{\gamma}_{k} \in \widetilde{h}_{k}} \widetilde{\gamma}_{k}^{U}\right.\right.\right.\right. \\
& \left.-\frac{1}{\# \widetilde{h}_{j}} \sum_{\tilde{\gamma}_{j} \in \widetilde{h}_{j}} \tilde{\gamma}_{j}^{L}\right) \\
& \times\left(\frac{1}{\# \widetilde{h}_{j}} \sum_{\tilde{\gamma}_{j} \in \tilde{h}_{j}}\left(\widetilde{\gamma}_{j}^{U}-\tilde{\gamma}_{j}^{L}\right)\right. \\
& +\frac{1}{\# \widetilde{h}_{k}} \\
& \left.\left.\left.\times \underset{\widetilde{\gamma}_{k} \in \tilde{h}_{k}}{ } \sum\left(\widetilde{\gamma}_{k}^{U}-\widetilde{\gamma}_{k}^{L}\right)\right)^{-1}\right), 0\right\} \\
& \left.+\frac{n}{2}-1\right)
\end{aligned}
$$

Definition 7 (see [28]). An ordered weighted averaging (OWA) operator of dimensions $n$ is a mapping OWA $: R^{n} \rightarrow$ $R$ that has an associated weight vector $w=\left(w_{1}, w_{2}, \ldots, w_{n}\right)^{T}$ with the properties $0 \leq w_{j} \leq 1(j=1,2, \ldots, n)$ and $\sum_{j=1}^{n} w_{j}=$ 1 , such that

$$
\operatorname{OWA}\left(a_{1}, a_{2}, \ldots, a_{n}\right)=w_{1} a_{\sigma(1)}+w_{2} a_{\sigma(2)}+\cdots+w_{n} a_{\sigma(1)},
$$

where $\sigma$ defines a permutation of $\{1,2, \ldots, n\}$ such that $a_{\sigma(j)} \geq$ $a_{\sigma(j+1)}$ for all $j$.

It is well known that the weights in OWA operator are assigned by the positions of argument variables, that is, there is a one-to-one relative relation for each associated weight and its corresponding value of argument variable $[4,29,30]$. Thus, we can find a permutation $\rho:\{1,2, \ldots, n\} \rightarrow\{1,2, \ldots, n\}$, which is the inverse permutation of $\sigma$; that is, $\rho=\sigma^{-1}$, and the OWA operator can be alternatively defined as

$$
\operatorname{OWA}^{\prime}\left(a_{1}, a_{2}, \ldots, a_{n}\right)=w_{\rho(1)} a_{1}+w_{\rho(2)} a_{2}+\cdots+w_{\rho(n)} a_{n},
$$

where $\rho=\sigma^{-1}:\{1,2, \ldots, n\} \rightarrow\{1,2, \ldots, n\}$ is the inverse permutation of $\sigma, a_{j}$ is the $\rho(i)$ th largest element of the collection of $a_{j}(i=1,2, \ldots, n)$, and $w=\left(w_{1}, w_{2}, \ldots, w_{n}\right)^{T}$ is the associated weight vector, with $w_{i} \in[0,1]$ and $\sum_{i=1}^{n} w_{i}=1$. 
Theorem 8. $w=\left(w_{1}, w_{2}, \ldots, w_{n}\right)^{T}$ is the associated weight vector with $w_{i} \in[0,1], \sum_{i=1}^{n} w_{i}=1$ and $\rho(\cdot)$ and $\sigma(\cdot)$ are two permutations of $\{1,2, \ldots, n\}$, if $\rho(\cdot)=\sigma(\cdot)^{-1}$; then

$$
\operatorname{OWA}\left(a_{1}, a_{2}, \ldots, a_{n}\right)=\operatorname{OWA}^{\prime}\left(a_{1}, a_{2}, \ldots, a_{n}\right)
$$

Proof. Suppose

$$
\begin{aligned}
\rho: & \operatorname{OWA}\left(a_{1}, a_{2}, \ldots, a_{n}\right) \\
= & w_{\rho(1)} a_{\rho(\sigma(1))}+w_{\rho(2)} a_{\rho(\sigma(2))} \\
& +\cdots+w_{\rho(n)} a_{\rho(\sigma(n))} \\
= & w_{\rho(1)} a_{1}+w_{\rho(2)} a_{2}+\cdots+w_{\rho(n)} a_{n} \\
= & \operatorname{OWA}^{\prime}\left(a_{1}, a_{2}, \ldots, a_{n}\right), \\
\sigma: & \operatorname{OWA}^{\prime}\left(a_{1}, a_{2}, \ldots, a_{n}\right) \\
= & w_{\sigma(\rho(1))} a_{\sigma(1)}+w_{\sigma(\rho(2))} a_{\sigma(2)} \\
& +\cdots+w_{\sigma(\rho(n))} a_{\sigma(n)} \\
= & w_{1} a_{\sigma(1)}+w_{2} a_{\sigma(2)}+\cdots+w_{n} a_{\sigma(1)} \\
= & \operatorname{OWA}\left(a_{1}, a_{2}, \ldots, a_{n}\right) .
\end{aligned}
$$

Hence, $\operatorname{OWA}\left(a_{1}, a_{2}, \ldots, a_{n}\right)=\operatorname{OWA}^{\prime}\left(a_{1}, a_{2}, \ldots, a_{n}\right)$.

\section{Interval-Valued Hesitant Fuzzy Hamacher Synergetic Weighted Aggregation Operators}

The weighted averaging operator and the ordered weighted averaging operator are the most common and basic aggregation operators. In the section, based on the above Hamacher operations of IVHFSs, we first develop the interval-valued hesitant fuzzy Hamacher weighted averaging (IVHFHWA) operator and the interval-valued hesitant fuzzy Hamacher ordered weighted averaging (IVHFHOWA) operator; then, based on which, we further propose the interval-valued hesitant fuzzy Hamacher synergetic weighted averaging (IVHFHSWA) operator to unify the IVHFHWA and IVHFHOWA operators. Furthermore, based on the geometric mean, we propose the interval-valued hesitant fuzzy Hamacher synergetic weighted geometric (IVHFHSWG) operators. The essential properties of the operators are studied and special cases are discussed.

\subsection{Interval-Valued Hesitant Fuzzy Hamacher Synergetic}

\section{Weighted Averaging Operator}

Definition 9. Let $\widetilde{h}_{j}=\bigcup_{\widetilde{\gamma}_{j} \in \widetilde{h}_{j}}\left\{\tilde{\gamma}_{j}=\left[\tilde{\gamma}_{j}^{L}, \widetilde{\gamma}_{j}^{U}\right]\right\}(j=1,2, \ldots, n)$ be a collection of IVHFEs, and $\omega=\left(\omega_{1}, \omega_{2}, \ldots, \omega_{n}\right)^{T}$ is the relative weighting vector of $\widetilde{h}_{j}(i=1,2, \ldots, n)$, with $\omega_{i} \in$ $[0,1]$ and $\sum_{i=1}^{n} \omega_{i}=1$. Then an interval-valued hesitant fuzzy Hamacher weighted averaging (IVHFHWA) operator is a mapping IVHFWA Hamacher $: \widetilde{H}^{n} \rightarrow \widetilde{H}$ such that

$$
\begin{aligned}
& \underset{\text { Hamacher }}{\operatorname{IVHFWA}}\left(\tilde{h}_{1}, \tilde{h}_{2}, \ldots, \tilde{h}_{n}\right) \\
& =\bigoplus_{j=1}^{n} \omega_{j} \tilde{h}_{j} \\
& =\underset{\widetilde{\gamma}_{j} \in \tilde{h}_{j}, j=1, \ldots, n}{\bigcup}\left\{\left[\left(\prod_{j=1}^{n}\left(1+(\eta-1) \tilde{\gamma}_{j}^{L}\right)^{\omega_{j}}\right.\right.\right. \\
& \left.-\prod_{j=1}^{n}\left(1-\tilde{\gamma}_{j}^{L}\right)^{\omega_{j}}\right) \\
& \times\left(\prod_{j=1}^{n}\left(1+(\eta-1) \tilde{\gamma}_{j}^{L}\right)^{\omega_{j}}\right. \\
& \left.+(\eta-1) \prod_{j=1}^{n}\left(1-\tilde{\gamma}_{j}^{L}\right)^{\omega_{j}}\right)^{-1} \\
& \left(\prod_{j=1}^{n}\left(1+(\eta-1) \widetilde{\gamma}_{j}^{U}\right)^{\omega_{j}}\right. \\
& \left.-\prod_{j=1}^{n}\left(1-\widetilde{\gamma}_{j}^{U}\right)^{\omega_{j}}\right) \\
& \times\left(\prod_{j=1}^{n}\left(1+(\eta-1) \widetilde{\gamma}_{j}^{U}\right)^{\omega_{j}}\right. \\
& \left.\left.\left.+(\eta-1) \prod_{j=1}^{n}\left(1-\widetilde{\gamma}_{j}^{U}\right)^{\omega_{j}}\right)^{-1}\right]\right\} .
\end{aligned}
$$

Because the algebraic $t$-norms and Einstein $t$-norms are the special cases of the Hamacher $t$-norms, the following theorems hold.

Theorem 10. The IVHFWA operator proposed by Chen et al. [11] is a special case of the IVHFHWA operator; that is, if $\eta=1$, then

$$
\begin{aligned}
& \underset{\text { Hamacher }}{\operatorname{IVHFWA}}\left(\widetilde{h}_{1}, \widetilde{h}_{2}, \ldots, \widetilde{h}_{n}\right) \stackrel{\eta=1}{\longrightarrow} \operatorname{IVHFWA}\left(\widetilde{h}_{1}, \widetilde{h}_{2}, \ldots, \widetilde{h}_{n}\right) \\
& =\bigcup_{\widetilde{\gamma}_{j} \in \widetilde{h}_{j}, j=1, \ldots, n}\left\{\left[1-\prod_{j=1}^{n}\left(1-\widetilde{\gamma}_{j}^{L}\right)^{\omega_{j}}, 1-\prod_{j=1}^{n}\left(1-\widetilde{\gamma}_{j}^{U}\right)^{\omega_{j}}\right]\right\} .
\end{aligned}
$$


Proof. Suppose

$$
\begin{aligned}
& \underset{\text { Hamacher }}{\operatorname{IVHFWA}}\left(\widetilde{h}_{1}, \tilde{h}_{2}, \ldots, \tilde{h}_{n}\right) \\
& =\bigcup_{\tilde{\gamma}_{j} \in \tilde{h}_{j}, j=1, \ldots, n}\left\{\left[\left(\prod_{j=1}^{n}\left(1+(1-1) \tilde{\gamma}_{j}^{L}\right)^{\omega_{j}}\right.\right.\right. \\
& \left.-\prod_{j=1}^{n}\left(1-\tilde{\gamma}_{j}^{L}\right)^{\omega_{j}}\right) \\
& \times\left(\prod_{j=1}^{n}\left(1+(\eta-1) \tilde{\gamma}_{j}^{L}\right)^{\omega_{j}}\right. \\
& \left.+(1-1) \prod_{j=1}^{n}\left(1-\widetilde{\gamma}_{j}^{L}\right)^{\omega_{j}}\right)^{-1}, \\
& \left(\prod_{j=1}^{n}\left(1+(1-1) \widetilde{\gamma}_{j}^{U}\right)^{\omega_{j}}\right. \\
& \left.-\prod_{j=1}^{n}\left(1-\widetilde{\gamma}_{j}^{U}\right)^{\omega_{j}}\right) \\
& \times\left(\prod_{j=1}^{n}\left(1+(1-1) \widetilde{\gamma}_{j}^{U}\right)^{\omega_{j}}\right. \\
& \left.\left.\left.+(1-1) \prod_{j=1}^{n}\left(1-\widetilde{\gamma}_{j}^{U}\right)^{\omega_{j}}\right)^{-1}\right]\right\} \\
& =\bigcup_{\tilde{\gamma}_{j} \in \tilde{h}_{j}, j=1, \ldots, n}\left\{\left[1-\prod_{j=1}^{n}\left(1-\widetilde{\gamma}_{j}^{L}\right)^{\omega_{j}}, 1-\prod_{j=1}^{n}\left(1-\widetilde{\gamma}_{j}^{U}\right)^{\omega_{j}}\right]\right\} \\
& =\operatorname{IVHFWA}\left(\widetilde{h}_{1}, \widetilde{h}_{2}, \ldots, \widetilde{h}_{n}\right) \text {. }
\end{aligned}
$$

Thus, IVHFWA Hamacher $\left(\widetilde{h}_{1}, \tilde{h}_{2}, \ldots, \widetilde{h}_{n}\right) \stackrel{\eta=1}{\longrightarrow}$ IVHFWA $\left(\widetilde{h}_{1}, \widetilde{h}_{2}, \ldots, \widetilde{h}_{n}\right)$.

Theorem 11. The IVHFEWA operator proposed by Wei and Zhao [23] is a special case of the IVHFHWA operator; that is, if $\eta=2$, then

$$
\begin{array}{r}
\underset{\text { Hamacher }}{\operatorname{IVHFWA}}\left(\tilde{h}_{1}, \tilde{h}_{2}, \ldots, \tilde{h}_{n}\right) \stackrel{\eta=2}{\longrightarrow} \operatorname{IVHFEWA}\left(\tilde{h}_{1}, \tilde{h}_{2}, \ldots, \tilde{h}_{n}\right) \\
=\underset{\tilde{\gamma}_{j} \in \tilde{h}_{j}, j=1, \ldots, n}{\bigcup}\left\{\left[\frac{\prod_{j=1}^{n}\left(1+\tilde{\gamma}_{j}^{L}\right)^{\omega_{j}}-\prod_{j=1}^{n}\left(1-\tilde{\gamma}_{j}^{L}\right)^{\omega_{j}}}{\prod_{j=1}^{n}\left(1+\tilde{\gamma}_{j}^{L}\right)^{\omega_{j}}+\prod_{j=1}^{n}\left(1-\tilde{\gamma}_{j}^{L}\right)^{\omega_{j}}},\right.\right. \\
\\
\left.\left.\frac{\prod_{j=1}^{n}\left(1+\tilde{\gamma}_{j}^{U}\right)^{\omega_{j}}-\prod_{j=1}^{n}\left(1-\tilde{\gamma}_{j}^{U}\right)^{\omega_{j}}}{\prod_{j=1}^{n}\left(1+\tilde{\gamma}_{j}^{U}\right)^{\omega_{j}}+\prod_{j=1}^{n}\left(1-\tilde{\gamma}_{j}^{U}\right)^{\omega_{j}}}\right]\right\} .
\end{array}
$$

\section{Proof. Suppose}

$$
\begin{aligned}
& \underset{\text { Hamacher }}{\operatorname{IVHFWA}}\left(\widetilde{h}_{1}, \widetilde{h}_{2}, \ldots, \widetilde{h}_{n}\right) \\
& =\bigoplus_{j=1}^{n} \omega_{j} \tilde{h}_{j} \\
& =\bigcup_{\tilde{\gamma}_{j} \in \tilde{h}_{j}, j=1, \ldots, n}\left\{\left[\left(\prod_{j=1}^{n}\left(1+(2-1) \tilde{\gamma}_{j}^{L}\right)^{\omega_{j}}\right.\right.\right. \\
& \left.-\prod_{j=1}^{n}\left(1-\tilde{\gamma}_{j}^{L}\right)^{\omega_{j}}\right) \\
& \times\left(\prod_{j=1}^{n}\left(1+(2-1) \tilde{\gamma}_{j}^{L}\right)^{\omega_{j}}\right. \\
& \left.+(2-1) \prod_{j=1}^{n}\left(1-\tilde{\gamma}_{j}^{L}\right)^{\omega_{j}}\right)^{-1}, \\
& \left(\prod_{j=1}^{n}\left(1+(2-1) \widetilde{\gamma}_{j}^{U}\right)^{\omega_{j}}\right. \\
& \left.-\prod_{j=1}^{n}\left(1-\widetilde{\gamma}_{j}^{U}\right)^{\omega_{j}}\right) \\
& \times\left(\prod_{j=1}^{n}\left(1+(2-1) \widetilde{\gamma}_{j}^{U}\right)^{\omega_{j}}\right. \\
& \left.\left.\left.+(2-1) \prod_{j=1}^{n}\left(1-\widetilde{\gamma}_{j}^{U}\right)^{\omega_{j}}\right)^{-1}\right]\right\} \\
& =\bigcup_{\tilde{\gamma}_{j} \in \tilde{h}_{j}, j=1, \ldots, n}\left\{\left[\left(\prod_{j=1}^{n}\left(1+\tilde{\gamma}_{j}^{L}\right)^{\omega_{j}}-\prod_{j=1}^{n}\left(1-\tilde{\gamma}_{j}^{L}\right)^{\omega_{j}}\right)\right.\right.
\end{aligned}
$$$$
\times\left(\prod_{j=1}^{n}\left(1+\widetilde{\gamma}_{j}^{L}\right)^{\omega_{j}}+\prod_{j=1}^{n}\left(1-\widetilde{\gamma}_{j}^{L}\right)^{\omega_{j}}\right)^{-1},
$$$$
\left(\prod_{j=1}^{n}\left(1+\widetilde{\gamma}_{j}^{U}\right)^{\omega_{j}}-\prod_{j=1}^{n}\left(1-\widetilde{\gamma}_{j}^{U}\right)^{\omega_{j}}\right)
$$$$
\times\left(\prod_{j=1}^{n}\left(1+\widetilde{\gamma}_{j}^{U}\right)^{\omega_{j}}\right.
$$$$
\left.\left.\left.+\prod_{j=1}^{n}\left(1-\tilde{\gamma}_{j}^{U}\right)^{\omega_{j}}\right)^{-1}\right]\right\}
$$$$
=\operatorname{IVHFEWA}\left(\widetilde{h}_{1}, \widetilde{h}_{2}, \ldots, \widetilde{h}_{n}\right) \text {. }
$$ 
Thus, IVHFWA Hamacher $\left(\widetilde{h}_{1}, \widetilde{h}_{2}, \ldots, \widetilde{h}_{n}\right) \stackrel{\eta=2}{\longrightarrow}$ IVHFEWA $\left(\widetilde{h}_{1}, \widetilde{h}_{2}, \ldots, \widetilde{h}_{n}\right)$.

From the above analysis, we know that the IVHFWA operator [11] and the IVHFEWA operator [23] are the special cases of the IVHFHWA operator, and the IVHFHWA operator can provide more special cases by selecting different values of parameter $\eta$, which can provide more choices for the decision makers and considerably enhance or deteriorate the performance of aggregation. Thus, the IVHFHWA operator is more general and more flexible. Similar to the IVHFWA operator and the IVHFEWA operator, the IVHFHWA operator is also monotonic, bounded, and idempotent.

Example 12. Given the collection of IVHFEs, $\widetilde{h}_{1}=\{[0.2,0.4]$, $[0.5,0.7],[0.6,0.8]\}$ and $\widetilde{h}_{2}=\{[0.4,0.5],[0.7,0.8]\}$, the relative weights are $\omega_{1}=0.3, \omega_{2}=0.7$, and suppose that the $\eta=1$, then

$$
\begin{aligned}
& \underset{\text { Hamacher }}{\operatorname{IVHFWA}}\left(\widetilde{h}_{1}, \widetilde{h}_{2}\right) \\
& =\bigoplus_{j=1}^{2} \omega_{j} \widetilde{h}_{j} \\
& =\bigcup_{\widetilde{\gamma}_{j} \in \widetilde{h}_{j}, j=1,2}\left\{\left[1-\prod_{j=1}^{2}\left(1-\widetilde{\gamma}_{j}^{L}\right)^{\omega_{j}}, 1-\prod_{j=1}^{2}\left(1-\widetilde{\gamma}_{j}^{U}\right)^{\omega_{j}}\right]\right\} \\
& \times\left\{\left[1-(1-0.2)^{0.3}(1-0.4)^{0.7},\right.\right. \\
& \left.1-(1-0.4)^{0.3}(1-0.5)^{0.7}\right], \\
& {\left[1-(1-0.2)^{0.3}(1-0.7)^{0.7}\right. \text {, }} \\
& \left.1-(1-0.4)^{0.3}(1-0.8)^{0.7}\right], \\
& {\left[1-(1-0.5)^{0.3}(1-0.4)^{0.7}\right. \text {, }} \\
& \left.1-(1-0.7)^{0.3}(1-0.5)^{0.7}\right], \\
& {\left[1-(1-0.5)^{0.3}(1-0.7)^{0.7}\right. \text {, }} \\
& \left.1-(1-0.7)^{0.3}(1-0.8)^{0.7}\right], \\
& {\left[1-(1-0.6)^{0.3}(1-0.4)^{0.7}\right. \text {, }} \\
& \left.1-(1-0.8)^{0.3}(1-0.5)^{0.7}\right], \\
& {\left[1-(1-0.6)^{0.3}(1-0.7)^{0.7}\right. \text {, }} \\
& \left.\left.1-(1-0.8)^{0.3}(1-0.8)^{0.7}\right]\right\} \\
& =\{[0.3459,0.4719],[0.5974,0.7219] \text {, } \\
& \text { [0.4319, 0.5710], [0.6503, 0.7741], } \\
& [0.4687,0.6202],[0.673,0.8]\} \text {. }
\end{aligned}
$$

Definition 13. Let $\widetilde{h}_{j}=\bigcup_{\tilde{\gamma}_{j} \in \tilde{h}_{j}}\left\{\widetilde{\gamma}_{j}=\left[\widetilde{\gamma}_{j}^{L}, \widetilde{\gamma}_{j}^{U}\right]\right\}(j=1,2, \ldots, n)$ be a collection of IVHFEs, and $w=\left(w_{1}, w_{2}, \ldots, w_{n}\right)^{T}$ is the associated weighting vector of $\widetilde{h}_{j}(i=1,2, \ldots, n)$, with $w_{i} \in[0,1]$ and $\sum_{i=1}^{n} w_{i}=1$. Then an interval-valued hesitant fuzzy Hamacher ordered weighted averaging (IVHFHOWA) operator is a mapping IVHFOWA Hamacher $: \widetilde{H}^{n} \rightarrow \widetilde{H}$ such that

$$
\begin{aligned}
& \underset{\text { Hamacher }}{\operatorname{IVHFOWA}}\left(\widetilde{h}_{1}, \widetilde{h}_{2}, \ldots, \widetilde{h}_{n}\right) \\
& =\bigoplus_{j=1}^{n} w_{j} \widetilde{h}_{\sigma(j)} \\
& =\bigcup_{\tilde{\gamma}_{j} \in \widetilde{h}_{j}, j=1, \ldots, n}\left\{\left[\left(\prod_{j=1}^{n}\left(1+(\eta-1) \tilde{\gamma}_{\sigma(j)}^{L}\right)^{w_{j}}\right.\right.\right. \\
& \left.-\prod_{j=1}^{n}\left(1-\tilde{\gamma}_{\sigma(j)}^{L}\right)^{w_{j}}\right) \\
& \times\left(\prod_{j=1}^{n}\left(1+(\eta-1) \tilde{\gamma}_{\sigma(j)}^{L}\right)^{w_{j}}\right. \\
& \left.+(\eta-1) \prod_{j=1}^{n}\left(1-\widetilde{\gamma}_{\sigma(j)}^{L}\right)^{w_{j}}\right)^{-1}, \\
& \left(\prod_{j=1}^{n}\left(1+(\eta-1) \widetilde{\gamma}_{\sigma(j)}^{U}\right)^{w_{j}}\right. \\
& \left.-\prod_{j=1}^{n}\left(1-\widetilde{\gamma}_{\sigma(j)}^{U}\right)^{w_{j}}\right) \\
& \times\left(\prod_{j=1}^{n}\left(1+(\eta-1) \widetilde{\gamma}_{\sigma(j)}^{U}\right)^{w_{j}}\right. \\
& \left.\left.\left.+(\eta-1) \prod_{j=1}^{n}\left(1-\widetilde{\gamma}_{\sigma(j)}^{U}\right)^{w_{j}}\right)^{-1}\right]\right\},
\end{aligned}
$$

where $\rho:\{1,2, \ldots, n\} \rightarrow\{1,2, \ldots, n\}$ is a permutation function such that $\widetilde{h}_{\sigma(j)}$ is the $\sigma(j)$ th largest element of the collection of $\widetilde{h}_{j}(i=1,2, \ldots, n)$.

On the other hand,

$$
\begin{gathered}
\underset{\text { Hamacher }}{\operatorname{IVHFOWA}}\left(\widetilde{h}_{1}, \widetilde{h}_{2}, \ldots, \widetilde{h}_{n}\right) \\
=\bigoplus_{j=1}^{n} \omega_{p(j)} \widetilde{h}_{j}
\end{gathered}
$$




$$
\begin{aligned}
=\bigcup_{\widetilde{\gamma}_{j} \in \tilde{h}_{j}, j=1, \ldots, n}\{[ & \prod_{j=1}^{n}\left(1+(\eta-1) \tilde{\gamma}_{j}^{L}\right)^{w_{\rho(j)}} \\
& \left.-\prod_{j=1}^{n}\left(1-\tilde{\gamma}_{j}^{L}\right)^{w_{\rho(j)}}\right) \\
& \times\left(\prod_{j=1}^{n}\left(1+(\eta-1) \tilde{\gamma}_{j}^{L}\right)^{w_{\rho(j)}}\right. \\
& \\
& \left(\prod_{j=1}^{n}\left(1+(\eta-1) \tilde{\gamma}_{j}^{U}\right)^{w_{\rho(j)}}\right. \\
& \times\left(\prod_{j=1}^{n}\left(1+(\eta-1) \prod_{j=1}^{n}\left(1-\widetilde{\gamma}_{j}^{L}\right)^{w_{\rho(j)}}\right)^{-1}\right. \\
& \left.-\prod_{j=1}^{n}\left(1-\widetilde{\gamma}_{j}^{U}\right)^{w_{\rho(j)}}\right)^{w_{\rho(j)}} \\
& \left.\left.\left.+(\eta-1) \prod_{j=1}^{n}\left(1-\widetilde{\gamma}_{j}^{U}\right)^{w_{\rho(j)}}\right)^{-1}\right]\right\}
\end{aligned}
$$

where $\rho:\{1,2, \ldots, n\} \rightarrow\{1,2, \ldots, n\}$ is a permutation function such that $\widetilde{h}_{j}$ is the $\rho(j)$ th largest element of the collection of $\widetilde{h}_{j}(i=1,2, \ldots, n)$.

Analogously, because the algebraic $t$-norms and Einstein $t$-norms are the special cases of the Hamacher $t$-norms, the following theorems hold.

Theorem 14. The IVHFOWA operator proposed by Chen et al. [11] is a special case of the IVHFHOWA operator; that is, if $\eta=$ 1 , then

$$
\begin{gathered}
\underset{\text { Hamacher }}{\operatorname{IVHFOWA}}\left(\widetilde{h}_{1}, \widetilde{h}_{2}, \ldots, \widetilde{h}_{n}\right) \stackrel{\eta=1}{\longrightarrow} \operatorname{IVHFOWA}\left(\widetilde{h}_{1}, \widetilde{h}_{2}, \ldots, \widetilde{h}_{n}\right) \\
=\bigcup_{\widetilde{\gamma}_{j} \in \tilde{h}_{j}, j=1, \ldots, n}\left\{\left[1-\prod_{j=1}^{n}\left(1-\widetilde{\gamma}_{\sigma(j)}^{L}\right)^{w_{j}},\right.\right. \\
\left.\left.1-\prod_{j=1}^{n}\left(1-\widetilde{\gamma}_{\sigma(j)}^{U}\right)^{w_{j}}\right]\right\} .
\end{gathered}
$$

Theorem 15. The IVHFEOWA operator proposed by Wei and Zhao [23] is a special case of the IVHFHOWA operator that is, if $\eta=2$, then

$$
\begin{aligned}
& \underset{\text { Hamacher }}{\operatorname{IVHFOWA}}\left(\widetilde{h}_{1}, \tilde{h}_{2}, \ldots, \widetilde{h}_{n}\right) \\
& \stackrel{\eta=2}{\longrightarrow} \operatorname{IVHFEOWA}\left(\widetilde{h}_{1}, \widetilde{h}_{2}, \ldots, \tilde{h}_{n}\right) \\
& =\bigcup_{\tilde{\gamma}_{j} \in \tilde{h}_{j}, j=1, \ldots, n}\left\{\left[\left(\prod_{j=1}^{n}\left(1+\widetilde{\gamma}_{\sigma(j)}^{L}\right)^{w_{j}}\right.\right.\right. \\
& \left.-\prod_{j=1}^{n}\left(1-\widetilde{\gamma}_{\sigma(j)}^{L}\right)^{w_{j}}\right) \\
& \times\left(\prod_{j=1}^{n}\left(1+\tilde{\gamma}_{\sigma(j)}^{L}\right)^{w_{j}}\right. \\
& \left.+\prod_{j=1}^{n}\left(1-\widetilde{\gamma}_{\sigma(j)}^{L}\right)^{w_{j}}\right)^{-1}, \\
& \left(\prod_{j=1}^{n}\left(1+\widetilde{\gamma}_{\sigma(j)}^{U}\right)^{w_{j}}\right. \\
& \left.-\prod_{j=1}^{n}\left(1-\widetilde{\gamma}_{\sigma(j)}^{U}\right)^{w_{j}}\right) \\
& \times\left(\prod_{j=1}^{n}\left(1+\widetilde{\gamma}_{\sigma(j)}^{U}\right)^{w_{j}}\right. \\
& \left.\left.\left.+\prod_{j=1}^{n}\left(1-\widetilde{\gamma}_{\sigma(j)}^{U}\right)^{w_{j}}\right)^{-1}\right]\right\} .
\end{aligned}
$$

Analogously, compared with IVHFOWA operator [11] and the IVHFEOWA operator [23], the IVHFHOWA operator can provide more special cases by selecting different values of parameter $\eta$, which can provide more choices for the decision makers and considerably enhance or deteriorate the performance of aggregation. Thus, the IVHFHOWA operator is more general and more flexible. Similar to the IVHFOWA operator and the IVHFEOWA operator, the IVHFHOWA operator is also commutative, monotonic, bounded, and idempotent.

Example 16. Given the collection of IVHFEs, $\tilde{h}_{1}=$ $\{[0.2,0.4],[0.5,0.7],[0.6,0.8]\}$ and $\widetilde{h}_{2}=\{[0.4,0.5],[0.7,0.8]\}$, the associated weights are $w_{1}=0.6$ and $w_{2}=0.4$, and suppose that the $\eta=1$, then, since

$$
\begin{aligned}
& P\left(\widetilde{h}_{1} \geq \widetilde{h}_{2}\right) \\
& =\max \left\{1-\max \left(\frac{(1 / 2)(0.5+0.8)-(1 / 3)(0.2+0.5+0.6)}{(1 / 3)(0.2+0.5+0.6)+(1 / 2)(0.1+0.1)},\right.\right. \\
& 0), 0\}=0.267,
\end{aligned}
$$


we have $\widetilde{h}_{1}<\widetilde{h}_{2}$ and the assigned associated weights of $\widetilde{h}_{1}$ and $\widetilde{h}_{2}$ are $w_{\rho(1)}=0.4$ and $w_{\rho(2)}=0.6$, respectively. Then

$$
\begin{aligned}
& \underset{\text { Hamacher }}{\operatorname{IVHFOWA}}\left(\widetilde{h}_{1}, \widetilde{h}_{2}\right) \\
& =\bigoplus_{j=1}^{2} w_{\rho(j)} \widetilde{h}_{j} \\
& =\bigcup_{\widetilde{\gamma}_{j} \in \widetilde{h}_{j}, j=1,2}\left\{\left[1-\prod_{j=1}^{2}\left(1-\tilde{\gamma}_{j}^{L}\right)^{w_{\rho(j)}},\right.\right. \\
& \left.\left.1-\prod_{j=1}^{2}\left(1-\widetilde{\gamma}_{j}^{U}\right)^{w_{\rho(j)}}\right]\right\} \\
& =\left\{\left[1-(1-0.2)^{0.4}(1-0.4)^{0.6}\right.\right. \text {, } \\
& \left.1-(1-0.4)^{0.4}(1-0.5)^{0.6}\right] \text {, } \\
& {\left[1-(1-0.2)^{0.4}(1-0.7)^{0.6}\right. \text {, }} \\
& \left.1-(1-0.4)^{0.4}(1-0.8)^{0.6}\right], \\
& {\left[1-(1-0.5)^{0.4}(1-0.4)^{0.6}\right. \text {, }} \\
& \left.1-(1-0.7)^{0.4}(1-0.5)^{0.6}\right], \\
& {\left[1-(1-0.5)^{0.4}(1-0.7)^{0.6}\right. \text {, }} \\
& \left.1-(1-0.7)^{0.4}(1-0.8)^{0.6}\right], \\
& {\left[1-(1-0.6)^{0.4}(1-0.4)^{0.6}\right. \text {, }} \\
& \left.1-(1-0.8)^{0.4}(1-0.5)^{0.6}\right], \\
& {\left[1-(1-0.6)^{0.4}(1-0.7)^{0.6}\right. \text {, }} \\
& \left.\left.1-(1-0.8)^{0.4}(1-0.8)^{0.6}\right]\right\} \\
& =\{[0.3268,0.4622],[0.5559,0.6896] \text {, } \\
& {[0.4422,0.5924],[0.6320,0.7648] \text {, }} \\
& [0.4898,0.6534],[0.6634,0.8]\} \text {. }
\end{aligned}
$$

According to the above analysis, we know that the IVHFHWA operator weights only the interval-valued hesitant fuzzy argument variables; its weights are the relative weights and represent the differential importance (salience, significance) of argument variables themselves, while the IVHFHOWA operator weights only the ordered positions of the intervalvalued hesitant fuzzy argument variables (or the magnitudes of the interval-valued hesitant fuzzy argument values); its weights are the associated weights and depend on the corresponding satisfaction values of argument variables. According to the associated weights are derived in view of the satisfaction values of argument variables; the IVHFHOWA operator can relieve (or intensify) the influence of unduly large or unduly small deviations on the aggregation results [31] or decide the portion of the criteria they feel is necessary for a good solution $[28,32,33]$. Therefore, weights represent different aspects in both the IVHFHWA and IVHFHOWA operators. However, in general, we need to consider the two weights because they represent different aspects of decision making problems. Obviously, both the IVHFHWA and IVHFHOWA operators have drawbacks. In order to solve these drawbacks, according to Theorem 8 and inspired by the idea of twofold weighting $[4,34]$, we propose an interval-valued hesitant fuzzy Hamacher synergetic weighted averaging (IVHFHSWA) operator in what follows.

Definition 17. Let $\widetilde{h}_{j}=\bigcup_{\widetilde{\gamma}_{j} \in \tilde{h}_{j}}\left\{\widetilde{\gamma}_{j}=\left[\tilde{\gamma}_{j}^{L}, \widetilde{\gamma}_{j}^{U}\right]\right\}(j=1,2, \ldots, n)$ be a collection of IVHFEs, and $\omega=\left(\omega_{1}, \omega_{2}, \ldots, \omega_{n}\right)^{T}$ is the relative weighting vector of the $\widetilde{h}_{j}(i=1,2, \ldots, n)$, with $\omega_{i} \in[0,1]$ and $\sum_{i=1}^{n} \omega_{i}=1$. Then an interval-valued hesitant fuzzy Hamacher synergetic weighted averaging (IVHFHSWA) operator is a mapping IVHFSWA Hamacher $: \widetilde{H}^{n} \rightarrow \widetilde{H}$, associated with a weighting vector $w=\left(w_{1}, w_{2}, \ldots, w_{n}\right)$, such that $w_{i} \in[0,1]$ and $\sum_{i=1}^{n} w_{i}=1$, according to the following expression:

$$
\begin{aligned}
& \operatorname{IVHFSWA}\left(\widetilde{h}_{1}, \widetilde{h}_{2}, \ldots, \widetilde{h}_{n}\right) \\
& =\frac{\bigoplus_{j=1}^{n} \omega_{j} \widetilde{h}_{j} w_{\rho(j)}}{\sum_{j=1}^{n} \omega_{j} w_{\rho(j)}} \\
& =\bigcup_{\tilde{\gamma}_{j} \in \tilde{h}_{j}, j=1, \ldots, n}\left\{\left[\left(\prod_{j=1}^{n}\left(1+(\eta-1) \tilde{\gamma}_{j}^{L}\right)^{\omega_{j} w_{\rho(j)} / \sum_{j=1}^{n} \omega_{j} w_{\rho(j)}}\right.\right.\right. \\
& \left.-\prod_{j=1}^{n}\left(1-\widetilde{\gamma}_{j}^{L}\right)^{\omega_{j} w_{\rho(j)} / \sum_{j=1}^{n} \omega_{j} \omega_{\rho(j)}}\right) \\
& \times\left(\prod_{j=1}^{n}\left(1+(\eta-1) \tilde{\gamma}_{j}^{L}\right)^{\omega_{j} w_{\rho(j)} / \sum_{j=1}^{n} \omega_{j} w_{\rho(j)}}\right. \\
& +(\eta-1) \\
& \left.\times \prod_{j=1}^{n}\left(1-\tilde{\gamma}_{j}^{L}\right)^{\omega_{j} w_{\rho(j)} / \sum_{j=1}^{n} \omega_{j} w_{\rho(j)}}\right)^{-1}, \\
& \left(\prod_{j=1}^{n}\left(1+(\eta-1) \widetilde{\gamma}_{j}^{U}\right)^{\omega_{j} w_{\rho(j)} / \sum_{j=1}^{n} \omega_{j} \omega_{\rho(j)}}\right. \\
& \left.-\prod_{j=1}^{n}\left(1-\widetilde{\gamma}_{j}^{U}\right)^{\omega_{j} w_{\rho(j)} / \sum_{j=1}^{n} \omega_{j} w_{\rho(j)}}\right) \\
& \times\left(\prod_{j=1}^{n}\left(1+(\eta-1) \widetilde{\gamma}_{j}^{U}\right)^{\omega_{j} w_{\rho(j)} / \sum_{j=1}^{n} \omega_{j} \omega_{\rho(j)}}\right. \\
& +(\eta-1) \\
& \left.\left.\left.\times \prod_{j=1}^{n}\left(1-\widetilde{\gamma}_{j}^{U}\right)^{\omega_{j} \omega_{\rho(j)} / \sum_{j=1}^{n} \omega_{j} \omega_{\rho(j)}}\right)^{-1}\right]\right\},
\end{aligned}
$$


where $\rho:\{1,2, \ldots, n\} \rightarrow\{1,2, \ldots, n\}$ is a permutation function such that $\widetilde{h}_{j}$ is the $\rho(j)$ th largest element of the collection of $\widetilde{h}_{j}(i=1,2, \ldots, n)$. In particular, if all $\widetilde{\gamma}_{j}^{L}=\widetilde{\gamma}_{j}^{U}$, the IVHFEs $\widetilde{h}_{j}=\bigcup_{\widetilde{\gamma}_{j} \in \tilde{h}_{j}}\left\{\widetilde{\gamma}_{j}=\left[\widetilde{\gamma}_{j}^{L}, \widetilde{\gamma}_{j}^{U}\right]\right\}(j=1,2, \ldots, n)$ are reduced to HFEs $h_{j}=\bigcup_{\gamma_{j} \in h_{j}}\left\{\gamma_{j}\right\}(j=1,2, \ldots, n)$, then the IVHFHSWA operator becomes a hesitant fuzzy Hamacher synergetic weighted averaging (HFHSWA) operator:

$$
\begin{aligned}
& \underset{\text { Hamacher }}{\operatorname{HFSW}}\left(h_{1}, h_{2}, \ldots, h_{n}\right) \\
& =\frac{\bigoplus_{j=1}^{n} \omega_{j} h_{j} w_{\rho(j)}}{\sum_{j=1}^{n} \omega_{j} w_{\rho(j)}} \\
& =\bigcup_{\gamma_{j} \in h_{j}, j=1, \ldots, n}\left\{\left(\prod_{j=1}^{n}\left(1+(\eta-1) \gamma_{j}\right)^{\omega_{j} w_{\rho(j)} / \sum_{j=1}^{n} \omega_{j} w_{\rho(j)}}\right.\right. \\
& \left.\quad-\prod_{j=1}^{n}\left(1-\gamma_{j}\right)^{\left.\omega_{j} w_{\rho(j)} / \sum_{j=1}^{n} \omega_{j} w_{\rho(j)}\right)}\right) \\
& \quad\left(\prod_{j=1}^{n}\left(1+(\eta-1) \gamma_{j}\right)^{\omega_{j} w_{\rho(j)} / \sum_{j=1}^{n} \omega_{j} w_{\rho(j)}}\right. \\
& \quad+(\eta-1) \\
& \left.\left.\quad \times \prod_{j=1}^{n}\left(1-\gamma_{j}\right)^{\omega_{j} w_{\rho(j)} / \sum_{j=1}^{n} \omega_{j} w_{\rho(j)}}\right)^{-1}\right\} .
\end{aligned}
$$

Example 18. Given the collection of IVHFEs, $\widetilde{h}_{1}=$ $\{[0.2,0.4],[0.5,0.7],[0.6,0.8]\}$ and $\widetilde{h}_{2}=\{[0.4,0.5],[0.7,0.8]\}$, the relative weights are $\omega_{1}=0.3, \omega_{2}=0.7$ and the associated weights are $w_{1}=0.6$ and $w_{2}=0.4$, and suppose that the $\eta=1$, then since

$$
\begin{aligned}
& P\left(\widetilde{h}_{1} \geq \widetilde{h}_{2}\right) \\
& =\max \left\{1-\max \left(\frac{(1 / 2)(0.5+0.8)-(1 / 3)(0.2+0.5+0.6)}{(1 / 3)(0.2+0.2+0.2)+(1 / 2)(0.1+0.1)},\right.\right. \\
& 0), 0\}=0.267,
\end{aligned}
$$

we have $\widetilde{h}_{1}<\widetilde{h}_{2}$, and the associated weights of $\widetilde{h}_{1}$ and $\widetilde{h}_{2}$ are $w_{\rho(1)}=0.4$ and $w_{\rho(2)}=0.6$, respectively. Thus

$$
\begin{aligned}
& \underset{\text { Hamacher }}{\operatorname{IVHFSWA}}\left(\widetilde{h}_{1}, \widetilde{h}_{2}\right) \\
& =\bigoplus_{j=1}^{2} \omega_{j} \widetilde{h}_{j} \\
& =\bigcup_{\tilde{\gamma}_{j} \in \widetilde{h}_{j}, j=1,2}\left\{\left[1-\prod_{j=1}^{2}\left(1-\widetilde{\gamma}_{j}^{L}\right)^{\omega_{j} \omega_{\rho(j)} / \sum_{j=1}^{2} \omega_{j} \omega_{\rho(j)},}\right.\right. \\
& \left.\left.1-\prod_{j=1}^{2}\left(1-\widetilde{\gamma}_{j}^{U}\right)^{\omega_{j} \omega_{\rho(j)} / \sum_{j=1}^{2} \omega_{j} \omega_{\rho(j)}}\right]\right\}
\end{aligned}
$$

$$
\begin{gathered}
=\left\{\left[1-(1-0.2)^{0.22}(1-0.4)^{0.78},\right.\right. \\
\left.1-(1-0.4)^{0.22}(1-0.5)^{0.78}\right], \\
{\left[1-(1-0.2)^{0.22}(1-0.7)^{0.78},\right.} \\
\left.1-(1-0.4)^{0.22}(1-0.8)^{0.78}\right], \\
{\left[1-(1-0.5)^{0.22}(1-0.4)^{0.78},\right.} \\
\left.1-(1-0.7)^{0.22}(1-0.5)^{0.78}\right], \\
{\left[1-(1-0.5)^{0.22}(1-0.7)^{0.78},\right.} \\
\left.1-(1-0.7)^{0.22}(1-0.8)^{0.78}\right], \\
{\left[1-(1-0.6)^{0.22}(1-0.4)^{0.78},\right.} \\
\left.1-(1-0.8)^{0.22}(1-0.5)^{0.78}\right], \\
{\left[1-(1-0.6)^{0.22}(1-0.7)^{0.78},\right.} \\
\left.\left.1-(1-0.8)^{0.22}(1-0.8)^{0.78}\right]\right\} \\
=\{[0.3608,0.4795],[0.6278,0.7453], \\
{[0.4236,0.5531],[0.6643,0.7813],} \\
[0.4512,0.5913],[0.6804,0.8]\} .
\end{gathered}
$$

By comparing Examples 12, 16, and 18, we know that the results derived from the IVHFHWA, IVHFHOWA, and IVHFHSWA operators are different; the reason is intuitive; the IVHFHWA operator focuses solely on the relative weights and ignores the associated weights in the process of aggregation, while the IVHFHOWA operator focuses only on the associated weights and ignores the relative weights in the process of aggregation; the IVHFHSWA operator comprehensively and simultaneously considers the relative weights and the associated weights and then its aggregated results are more feasible and effective.

Inevitably, we can see that the aggregation procedure of the interval-valued hesitant fuzzy information is complex, especially, with the increases of the number of argument variables, but it can surely better describe the situations where people have hesitancy in providing their preferences in the process of decision making, and the proposed operators can be easily solved using Microsoft Excel Solver or integrated in a decision support system $[4,10]$.

IVHFHSWA operator not only integrates the relative weights and the associated weights into the weighted averaging operation and then generalizes the IVHFHWA operator and IVHFHOWA operator but also satisfies the properties of idempotency, boundary and monotonicity, and so on.

Theorem 19 (Idempotency). Let $\tilde{h}_{j}=\bigcup_{\tilde{\gamma}_{j} \in \tilde{h}_{j}}\left\{\tilde{\gamma}_{j}=\right.$ $\left.\left[\widetilde{\gamma}_{j}^{L}, \widetilde{\gamma}_{j}^{U}\right]\right\}(j=1,2, \ldots, n)$ be a collection of IVHFEs; if $\widetilde{h}_{j}=$ $\widetilde{h}=\bigcup_{\tilde{\gamma} \in \tilde{h}}\left\{\tilde{\gamma}=\left[\widetilde{\gamma}^{L}, \widetilde{\gamma}^{U}\right]\right\}$ for all $j=1,2, \ldots, n$, then 
Proof. Consider

$$
\begin{aligned}
& \underset{\text { Hamacher }}{\operatorname{IVHFSWA}}\left(\widetilde{h}_{1}, \widetilde{h}_{2}, \ldots, \widetilde{h}_{n}\right) \\
& =\frac{\bigoplus_{j=1}^{n} \omega_{j} \widetilde{h}_{j} w_{\rho(j)}}{\sum_{j=1}^{n} \omega_{j} w_{\rho(j)}} \\
& =\bigcup_{\tilde{\gamma}_{j} \in \widetilde{h}_{j}, j=1, \ldots, n}\left\{\left[\left(\prod_{j=1}^{n}\left(1+(\eta-1) \tilde{\gamma}_{j}^{L}\right)^{\omega_{j} w_{\rho(j)} / \sum_{j=1}^{n} \omega_{j} w_{\rho(j)}}\right.\right.\right. \\
& \left.-\prod_{j=1}^{n}\left(1-\widetilde{\gamma}_{j}^{L}\right)^{\omega_{j} w_{\rho(j)} / \sum_{j=1}^{n} \omega_{j} w_{\rho(j)}}\right) \\
& \times\left(\prod_{j=1}^{n}\left(1+(\eta-1) \tilde{\gamma}_{j}^{L}\right)^{\omega_{j} w_{\rho(j)} / \sum_{j=1}^{n} \omega_{j} w_{\rho(j)}}\right. \\
& \left.+(\eta-1) \prod_{j=1}^{n}\left(1-\widetilde{\gamma}_{j}^{L}\right)^{\omega_{j} w_{\rho(j)} / \sum_{j=1}^{n} \omega_{j} w_{\rho(j)}}\right)^{-1}, \\
& \left(\prod_{j=1}^{n}\left(1+(\eta-1) \widetilde{\gamma}_{j}^{U}\right)^{\omega_{j} w_{\rho(j)} / \sum_{j=1}^{n} \omega_{j} w_{\rho(j)}}\right. \\
& \left.-\prod_{j=1}^{n}\left(1-\widetilde{\gamma}_{j}^{U}\right)^{\omega_{j} w_{\rho(j)} / \sum_{j=1}^{n} \omega_{j} w_{\rho(j)}}\right) \\
& \times\left(\prod_{j=1}^{n}\left(1+(\eta-1) \widetilde{\gamma}_{j}^{U}\right)^{\omega_{j} \omega_{\rho(j)} / \sum_{j=1}^{n} \omega_{j} w_{\rho(j)}}\right. \\
& +(\eta-1) \\
& \left.\left.\left.\times \prod_{j=1}^{n}\left(1-\widetilde{\gamma}_{j}^{U}\right)^{\omega_{j} w_{\rho(j)} / \sum_{j=1}^{n} \omega_{j} w_{\rho(j)}}\right)^{-1}\right]\right\} \\
& =\bigcup_{\widetilde{\gamma}_{j} \in \widetilde{h}_{j}, j=1, \ldots, n}\left\{\left[\left(\prod_{j=1}^{n}\left(1+(\eta-1) \tilde{\gamma}^{L}\right)^{\omega_{j} w_{\rho(j)} / \sum_{j=1}^{n} \omega_{j} w_{\rho(j)}}\right.\right.\right. \\
& \left.-\prod_{j=1}^{n}\left(1-\widetilde{\gamma}^{L}\right)^{\omega_{j} \omega_{\rho(j)} / \sum_{j=1}^{n} \omega_{j} w_{\rho(j)}}\right) \\
& \times\left(\prod_{j=1}^{n}\left(1+(\eta-1) \tilde{\gamma}^{L}\right)^{\omega_{j} \omega_{\rho(j)} / \sum_{j=1}^{n} \omega_{j} \omega_{\rho(j)}}\right. \\
& +(\eta-1) \\
& \left.\times \prod_{j=1}^{n}\left(1-\tilde{\gamma}^{L}\right)^{\omega_{j} \omega_{\rho(j)} / \sum_{j=1}^{n} \omega_{j} \omega_{\rho(j)}}\right)^{-1}, \\
& \left(\prod_{j=1}^{n}\left(1+(\eta-1) \widetilde{\gamma}^{U}\right)^{\omega_{j} \omega_{\rho(j)} / \sum_{j=1}^{n} \omega_{j} \omega_{\rho(j)}}\right.
\end{aligned}
$$

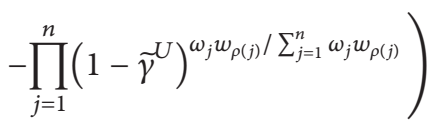

$$
\begin{array}{r}
\times\left(\prod_{j=1}^{n}\left(1+(\eta-1) \widetilde{\gamma}^{U}\right)^{\omega_{j} \omega_{\rho(j)} / \sum_{j=1}^{n} \omega_{j} \omega_{\rho(j)}}\right. \\
+(\eta-1) \\
\left.\left.\left.\times \prod_{j=1}^{n}\left(1-\widetilde{\gamma}^{U}\right)^{\omega_{j} \omega_{\rho(j)} / \sum_{j=1}^{n} \omega_{j} \omega_{\rho(j)}}\right)^{-1}\right]\right\} \\
=\bigcup_{\widetilde{\gamma}_{j} \in \tilde{h}_{j}, j=1,2, \ldots, n}\left\{\left[\frac{\left(1+(\eta-1) \tilde{\gamma}^{L}\right)-\left(1-\tilde{\gamma}^{L}\right)}{\left(1+(\eta-1) \tilde{\gamma}^{L}\right)+(\eta-1)\left(1-\widetilde{\gamma}^{L}\right)},\right.\right. \\
=\bigcup_{\tilde{\gamma}_{j} \in \tilde{h}_{j}, j=1, \ldots, n}\left\{\left[\widetilde{\gamma}^{L}, \widetilde{\gamma}^{U}\right]\right\}=\widetilde{h} .
\end{array}
$$

Thus, IVHFSWA $\mathrm{IHamacher}\left(\widetilde{h}_{1}, \widetilde{h}_{2}, \ldots, \widetilde{h}_{n}\right)=\widetilde{h}$.

Theorem 20 (Boundedness). Let $\tilde{h}_{j}=\bigcup_{\tilde{\gamma}_{j} \in \tilde{h}_{j}}\left\{\tilde{\gamma}_{j}=\right.$ $\left.\left[\widetilde{\gamma}_{j}^{L}, \widetilde{\gamma}_{j}^{U}\right]\right\}(j=1,2, \ldots, n)$ be a collection of IVHFEs, $\bigcap_{j=1}^{n} \widetilde{h}_{j}=$ $\bigcup_{\tilde{\gamma}_{j} \in \tilde{h}_{j}, j=1,2, \ldots, n}\left\{\left[\min _{1 \leq j \leq n}\left\{\tilde{\gamma}_{j}^{L}\right\}, \min _{1 \leq j \leq n}\left\{\tilde{\gamma}_{j}^{U}\right\}\right]\right\}$, and $\bigcup_{j=1}^{n} \widetilde{h}_{j}=$ $\bigcup_{\tilde{\gamma}_{j} \in \tilde{h}_{j}, j=1,2, \ldots, n}\left\{\left[\max _{1 \leq j \leq n}\left\{\tilde{\gamma}_{j}^{L}\right\}, \max _{1 \leq j \leq n}\left\{\widetilde{\gamma}_{j}^{U}\right\}\right]\right\} ;$ then

$$
\bigcap_{j=1}^{n} \widetilde{h}_{j} \leq \underset{\text { Hamacher }}{\operatorname{IVHSWA}}\left(\widetilde{h}_{1}, \widetilde{h}_{2}, \ldots, \widetilde{h}_{n}\right) \leq \bigcup_{j=1}^{n} \widetilde{h}_{j} .
$$

Proof. Suppose $\bigcap_{j=1}^{n} \tilde{h}_{j}=\bigcup_{\tilde{\gamma}_{j} \in \tilde{h}_{j}, j=1,2, \ldots, n}\left\{\left[\min _{1 \leq j \leq n}\left\{\tilde{\gamma}_{j}^{L}\right\}\right.\right.$, $\left.\left.\min _{1 \leq j \leq n}\left\{\widetilde{\gamma}_{j}^{U}\right\}\right]\right\}$, and

$$
\bigcup_{j=1}^{n} \widetilde{h}_{j}=\bigcup_{\tilde{\gamma}_{j} \in \widetilde{h}_{j}, j=1,2, \ldots, n}\left\{\left[\max _{1 \leq j \leq n}\left\{\widetilde{\gamma}_{j}^{L}\right\}, \max _{1 \leq j \leq n}\left\{\widetilde{\gamma}_{j}^{U}\right\}\right]\right\} .
$$

For any a combination, we always have

$$
\begin{aligned}
& {\left[\min _{1 \leq j \leq n}\left\{\widetilde{\gamma}_{j}^{L}\right\}, \min _{1 \leq j \leq n}\left\{\widetilde{\gamma}_{j}^{U}\right\}\right]} \\
& \leq\left[\left(\prod_{j=1}^{n}\left(1+(\eta-1) \widetilde{\gamma}_{j}^{L}\right)^{\omega_{j} w_{\rho(j)} / \sum_{j=1}^{n} \omega_{j} w_{\rho(j)}}\right.\right. \\
& \left.\quad-\prod_{j=1}^{n}\left(1-\widetilde{\gamma}_{j}^{L}\right)^{\omega_{j} w_{\rho(j)} / \sum_{j=1}^{n} \omega_{j} w_{\rho(j)}}\right) \\
& \times\left(\prod_{j=1}^{n}\left(1+(\eta-1) \tilde{\gamma}_{j}^{L}\right)^{\omega_{j} w_{\rho(j)} / \sum_{j=1}^{n} \omega_{j} \omega_{\rho(j)}}\right. \\
& \left.\quad+(\eta-1) \prod_{j=1}^{n}\left(1-\widetilde{\gamma}_{j}^{L}\right)^{\omega_{j} w_{\rho(j)} / \sum_{j=1}^{n} \omega_{j} w_{\rho(j)}}\right)^{-1},
\end{aligned}
$$




$$
\begin{aligned}
& \left(\prod_{j=1}^{n}\left(1+(\eta-1) \widetilde{\gamma}_{j}^{U}\right)^{\omega_{j} w_{\rho(j)} / \sum_{j=1}^{n} \omega_{j} w_{\rho(j)}}\right. \\
& \left.-\prod_{j=1}^{n}\left(1-\widetilde{\gamma}_{j}^{U}\right)^{\omega_{j} w_{\rho(j)} / \sum_{j=1}^{n} \omega_{j} w_{\rho(j)}}\right) \\
& \times\left(\prod_{j=1}^{n}\left(1+(\eta-1) \widetilde{\gamma}_{j}^{U}\right)^{\omega_{j} w_{\rho(j)} / \sum_{j=1}^{n} \omega_{j} w_{\rho(j)}}\right. \\
& \left.\left.+(\eta-1) \prod_{j=1}^{n}\left(1-\widetilde{\gamma}_{j}^{U}\right)^{\omega_{j} w_{\rho(j)} / \sum_{j=1}^{n} \omega_{j} w_{\rho(j)}}\right)^{-1}\right] \\
& \leq\left[\max _{1 \leq j \leq n}\left\{\widetilde{\gamma}_{j}^{L}\right\}, \max _{1 \leq j \leq n}\left\{\widetilde{\gamma}_{j}^{U}\right\}\right] .
\end{aligned}
$$

Then, considering all possible combinations, we have

$$
\begin{aligned}
& \bigcup_{\tilde{\gamma}_{j} \in \tilde{h}_{j}, j=1,2, \ldots, n}\left\{\left[\min _{1 \leq j \leq n}\left\{\tilde{\gamma}_{j}^{L}\right\}, \min _{1 \leq j \leq n}\left\{\widetilde{\gamma}_{j}^{U}\right\}\right]\right\} \\
& \leq \bigcup_{\widetilde{\gamma}_{j} \in \widetilde{h}_{j}, j=1,2, \ldots, n}\left\{\left[\left(\prod_{j=1}^{n}\left(1+(\eta-1) \tilde{\gamma}_{j}^{L}\right)^{\omega_{j} w_{\rho(j)} / \sum_{j=1}^{n} \omega_{j} \omega_{\rho(j)}}\right.\right.\right. \\
& \left.-\prod_{j=1}^{n}\left(1-\tilde{\gamma}_{j}^{L}\right)^{\omega_{j} w_{\rho(j)} / \sum_{j=1}^{n} \omega_{j} \omega_{\rho(j)}}\right) \\
& \times\left(\prod_{j=1}^{n}(1\right. \\
& \left.+(\eta-1) \tilde{\gamma}_{j}^{L}\right)^{\omega_{j} w_{\rho(j)} / \sum_{j=1}^{n} \omega_{j} w_{\rho(j)}} \\
& +(\eta-1) \\
& \left.\times \prod_{j=1}^{n}\left(1-\widetilde{\gamma}_{j}^{L}\right)^{\omega_{j} w_{\rho(j)} / \sum_{j=1}^{n} \omega_{j} w_{\rho(j)}}\right)^{-1} \\
& \bigcup_{\widetilde{\gamma}_{j} \in \widetilde{h}_{j}, j=1,2, \ldots, n}\left(\prod_{j=1}^{n}(1\right. \\
& \left.+(\eta-1) \widetilde{\gamma}_{j}^{U}\right)^{\omega_{j} w_{\rho(j)} / \sum_{j=1}^{n} \omega_{j} w_{\rho(j)}} \\
& \left.-\prod_{j=1}^{n}\left(1-\widetilde{\gamma}_{j}^{U}\right)^{\omega_{j} w_{\rho(j)} / \sum_{j=1}^{n} \omega_{j} w_{\rho(j)}}\right)
\end{aligned}
$$

$$
\begin{aligned}
& \times\left(\prod_{j=1}^{n}\left(1+(\eta-1) \widetilde{\gamma}_{j}^{U}\right)^{\omega_{j} \omega_{\rho(j)} / \sum_{j=1}^{n} \omega_{j} w_{\rho(j)}}\right. \\
&+(\eta-1) \\
&\left.\left.\left.\times \prod_{j=1}^{n}\left(1-\widetilde{\gamma}_{j}^{U}\right)^{\omega_{j} w_{\rho(j)} / \sum_{j=1}^{n} \omega_{j} w_{\rho(j)}}\right)^{-1}\right]\right\} \\
& \leq \bigcup_{\tilde{\gamma}_{j} \in \tilde{h}_{j}, j=1,2, \ldots, n}\left\{\left[\max _{1 \leq j \leq n}\left\{\tilde{\gamma}_{j}^{L}\right\}, \max _{1 \leq j \leq n}\left\{\widetilde{\gamma}_{j}^{U}\right\}\right]\right\} .
\end{aligned}
$$

Hence, we have $\bigcap_{j=1}^{n} \widetilde{h}_{j} \leq$ IVHFSWA $_{\text {Hamacher }}$ $\left(\widetilde{h}_{1}, \widetilde{h}_{2}, \ldots, \widetilde{h}_{n}\right) \leq \bigcup_{j=1}^{n} \widetilde{h}_{j}$.

Theorem 21 (Monotonicity). Let $\tilde{h}_{j}=\bigcup_{\tilde{\gamma}_{j} \in \tilde{h}_{j}}\left\{\tilde{\gamma}_{j}=\left[\tilde{\gamma}_{j}^{L}, \widetilde{\gamma}_{j}^{U}\right]\right\}$ and $\tilde{h}_{j}^{\prime}=\bigcup_{\tilde{\gamma}_{j}^{\prime} \tilde{h}_{j}^{\prime}}\left\{\tilde{\gamma}_{j}^{\prime}=\left[\tilde{\gamma}_{j}^{L}, \tilde{\gamma}_{j}^{U}\right]\right\}(j=1,2, \ldots, n)$ be two collections of IVHFEs; if only if $\widetilde{\gamma}_{j} \geq \widetilde{\gamma}_{j}^{\prime}, \widetilde{\gamma}_{j} \in \widetilde{h}_{j}$, and $\widetilde{\gamma}_{j}^{\prime} \in \widetilde{\gamma}_{j}^{\prime}$ for all $j=1,2, \ldots, n$, then

$$
\underset{\text { Hamacher }}{\operatorname{VHFSWA}}\left(h_{1}, h_{2}, \ldots, h_{n}\right) \geq \underset{\text { Hamacher }}{\operatorname{IVHFSWA}}\left(h_{1}^{\prime}, h_{2}^{\prime}, \ldots, h_{n}^{\prime}\right) .
$$

Proof. We have known that $\varphi_{\eta}(x, y)=(x+y-x y-(1-$ $\eta) x y) /(1-(1-\eta) x y), \eta>0$, is a strictly increasing function. Since $\widetilde{\gamma}_{j} \geq \widetilde{\gamma}_{j}^{\prime}, \widetilde{\gamma}_{j} \in \widetilde{h}_{j}, \widetilde{\gamma}_{j}^{\prime} \in \widetilde{h}_{j}^{\prime}$ for all $j=1,2, \ldots, n$, then

$$
\begin{aligned}
& {\left[\left(\prod_{j=1}^{n}\left(1+(\eta-1) \tilde{\gamma}_{j}^{L}\right)^{\omega_{j} w_{\rho(j)} / \sum_{j=1}^{n} \omega_{j} w_{\rho(j)}}\right.\right.} \\
& \left.\quad-\prod_{j=1}^{n}\left(1-\tilde{\gamma}_{j}^{L}\right)^{\omega_{j} w_{\rho(j)} / \sum_{j=1}^{n} \omega_{j} w_{\rho(j)}}\right) \\
& \quad \times\left(\prod_{j=1}^{n}\left(1+(\eta-1) \widetilde{\gamma}_{j}^{L}\right)^{\omega_{j} w_{\rho(j)} / \sum_{j=1}^{n} \omega_{j} w_{\rho(j)}}\right. \\
& \left.\quad+(\eta-1) \prod_{j=1}^{n}\left(1-\widetilde{\gamma}_{j}^{L}\right)^{\omega_{j} w_{\rho(j)} / \sum_{j=1}^{n} \omega_{j} w_{\rho(j)}}\right)^{-1}, \\
& \left(\prod_{j=1}^{n}\left(1+(\eta-1) \widetilde{\gamma}_{j}^{U}\right)^{\omega_{j} w_{\rho(j)} / \sum_{j=1}^{n} \omega_{j} w_{\rho(j)}}\right. \\
& \left.\quad-\prod_{j=1}^{n}\left(1-\widetilde{\gamma}_{j}^{U}\right)^{\omega_{j} w_{\rho(j)} / \sum_{j=1}^{n} \omega_{j} w_{\rho(j)}}\right)
\end{aligned}
$$




$$
\begin{aligned}
& \times\left(\prod_{j=1}^{n}\left(1+(\eta-1) \widetilde{\gamma}_{j}^{U}\right)^{\omega_{j} w_{\rho(j)} / \sum_{j=1}^{n} \omega_{j} w_{\rho(j)}}\right. \\
& \left.\left.+(\eta-1) \prod_{j=1}^{n}\left(1-\widetilde{\gamma}_{j}^{U}\right)^{\omega_{j} w_{\rho(j)} / \sum_{j=1}^{n} \omega_{j} w_{\rho(j)}}\right)^{-1}\right] \\
& \geq\left[\left(\prod_{j=1}^{n}\left(1+(\eta-1) \widetilde{\gamma}_{j}^{L}\right)^{\omega_{j} w_{\rho(j)} / \sum_{j=1}^{n} \omega_{j} w_{\rho(j)}}\right.\right. \\
& \left.-\prod_{j=1}^{n}\left(1-\widetilde{\gamma}_{j}^{L L}\right)^{\omega_{j} w_{\rho(j)} / \sum_{j=1}^{n} \omega_{j} w_{\rho(j)}}\right) \\
& \times\left(\prod_{j=1}^{n}\left(1+(\eta-1) \widetilde{\gamma}_{j}^{L}\right)^{\omega_{j} w_{\rho(j)} / \sum_{j=1}^{n} \omega_{j} w_{\rho(j)}}\right. \\
& \left.+(\eta-1) \prod_{j=1}^{n}\left(1-\widetilde{\gamma}_{j}^{L}\right)^{\omega_{j} w_{\rho(j)} / \sum_{j=1}^{n} \omega_{j} w_{\rho(j)}}\right)^{-1}, \\
& \left(\prod_{j=1}^{n}\left(1+(\eta-1) \widetilde{\gamma}_{j}^{\prime U}\right)^{\omega_{j} w_{\rho(j)} / \sum_{j=1}^{n} \omega_{j} w_{\rho(j)}}\right. \\
& \left.-\prod_{j=1}^{n}\left(1-\widetilde{\gamma}_{j}^{\prime U}\right)^{\omega_{j} w_{\rho(j)} / \sum_{j=1}^{n} \omega_{j} w_{\rho(j)}}\right) \\
& \times\left(\prod_{j=1}^{n}\left(1+(\eta-1) \widetilde{\gamma}_{j}^{\prime U}\right)^{\omega_{j} w_{\rho(j)} / \sum_{j=1}^{n} \omega_{j} w_{\rho(j)}}\right. \\
& \left.\left.+(\eta-1) \prod_{j=1}^{n}\left(1-\widetilde{\gamma}_{j}^{U}\right)^{\omega_{j} w_{\rho(j)} / \sum_{j=1}^{n} \omega_{j} w_{\rho(j)}}\right)^{-1}\right] .
\end{aligned}
$$

By considering all possible combinations, we can get easily that IVHFSWA Hamacher $\left(h_{1}, h_{2}, \ldots, h_{n}\right) \quad \geq$ IVHFSWA $_{\text {Hamacher }}\left(h_{1}^{\prime}, h_{2}^{\prime}, \ldots, h_{n}^{\prime}\right)$.

In the following, we discuss some special cases of the IVHFHSWA operator.

(1) From the perspective of parameter $\eta$.

Case 1. If $\eta=1$, then the IVHFHSWA operator results in an interval-valued hesitant fuzzy synergetic weighted averaging (IVHFSWA) operator:

$$
\begin{gathered}
\underset{\text { Hamacher }}{\operatorname{IVHFSWA}}\left(\widetilde{h}_{1}, \tilde{h}_{2}, \ldots, \tilde{h}_{n}\right) \stackrel{\eta=1}{\longrightarrow} \operatorname{IVHFSWA}\left(\widetilde{h}_{1}, \tilde{h}_{2}, \ldots, \widetilde{h}_{n}\right) \\
=\underset{\tilde{\gamma}_{j} \in \tilde{h}_{j}, j=1, \ldots, n}{\bigcup}\left\{\left[1-\prod_{j=1}^{n}\left(1-\widetilde{\gamma}_{j}^{L}\right)^{\omega_{j} w_{\rho(j)} / \sum_{j=1}^{n} \omega_{j} w_{\rho(j)}},\right.\right. \\
\left.\left.1-\prod_{j=1}^{n}\left(1-\widetilde{\gamma}_{j}^{U}\right)^{\omega_{j} w_{\rho(j)} / \sum_{j=1}^{n} \omega_{j} w_{\rho(j)}}\right]\right\} .
\end{gathered}
$$

Case 2. If $\eta=2$, then the IVHFHSWA operator results in an interval-valued hesitant fuzzy Einstein synergetic weighted averaging (IVHFESWA) operator:

$$
\begin{aligned}
& \underset{\text { Hamacher }}{\operatorname{IVHFSWA}}\left(\widetilde{h}_{1}, \widetilde{h}_{2}, \ldots, \widetilde{h}_{n}\right) \stackrel{\eta=2}{\longrightarrow} \operatorname{IVHFESWA}\left(\widetilde{h}_{1}, \widetilde{h}_{2}, \ldots, \widetilde{h}_{n}\right) \\
& =\bigcup_{\tilde{\gamma}_{j} \in \tilde{h}_{j}, j=1, \ldots, n}\left\{\left[\left(\prod_{j=1}^{n}\left(1+\widetilde{\gamma}_{j}^{L}\right)^{\omega_{j} w_{\rho(j)} / \sum_{j=1}^{n} \omega_{j} w_{\rho(j)}}\right.\right.\right. \\
& \left.-\prod_{j=1}^{n}\left(1-\tilde{\gamma}_{j}^{L}\right)^{\omega_{j} w_{\rho(j)} / \sum_{j=1}^{n} \omega_{j} w_{\rho(j)}}\right) \\
& \times\left(\prod_{j=1}^{n}\left(1+\widetilde{\gamma}_{j}^{L}\right)^{\omega_{j} w_{\rho(j)} / \sum_{j=1}^{n} \omega_{j} w_{\rho(j)}}\right. \\
& \left.+\prod_{j=1}^{n}\left(1-\widetilde{\gamma}_{j}^{L}\right)^{\omega_{j} w_{\rho(j)} / \sum_{j=1}^{n} \omega_{j} w_{\rho(j)}}\right)^{-1}, \\
& \left(\prod_{j=1}^{n}\left(1+\widetilde{\gamma}_{j}^{U}\right)^{\omega_{j} w_{\rho(j)} / \sum_{j=1}^{n} \omega_{j} w_{\rho(j)}}\right. \\
& \left.-\prod_{j=1}^{n}\left(1-\widetilde{\gamma}_{j}^{U}\right)^{\omega_{j} w_{\rho(j)} / \sum_{j=1}^{n} \omega_{j} w_{\rho(j)}}\right) \\
& \times\left(\prod_{j=1}^{n}\left(1+\widetilde{\gamma}_{j}^{U}\right)^{\omega_{j} w_{\rho(j)} / \sum_{j=1}^{n} \omega_{j} w_{\rho(j)}}\right. \\
& +\prod_{j=1}^{n}(1 \\
& \left.\left.\left.\left.-\widetilde{\gamma}_{j}^{U}\right)^{\omega_{j} w_{\rho(j)} / \sum_{j=1}^{n} \omega_{j} w_{\rho(j)}}\right)^{-1}\right]\right\} \text {. }
\end{aligned}
$$

(2) From the perspective of the types of weights.

Case 1. If the relative weighting vector $\omega=\left(\omega_{1}, \omega_{2}, \ldots, \omega_{n}\right)=$ $(1 / n, 1 / n, \ldots, 1 / n)$, then the IVHFHSWA operator is reduced to the IVHFHOWA operator.

Proof. Suppose

$$
\begin{aligned}
& \underset{\text { Hamacher }}{\operatorname{IVHFSWA}}\left(\widetilde{h}_{1}, \widetilde{h}_{2}, \ldots, \widetilde{h}_{n}\right) \\
& =\bigcup_{\tilde{\gamma}_{j} \in \tilde{h}_{j}, j=1, \ldots, n}\left\{\left[\left(\prod_{j=1}^{n}\left(1+(\eta-1) \tilde{\gamma}_{j}^{L}\right)^{(1 / n) w_{\rho(j)} / \sum_{j=1}^{n}(1 / n) w_{\rho(j)}}\right.\right.\right. \\
& \left.-\prod_{j=1}^{n}\left(1-\tilde{\gamma}_{j}^{L}\right)^{(1 / n) w_{\rho(j)} / \sum_{j=1}^{n}(1 / n) w_{\rho(j)}}\right) \\
& \times\left(\prod_{j=1}^{n}\left(1+(\eta-1) \tilde{\gamma}_{j}^{L}\right)^{(1 / n) w_{\rho(j)} / \sum_{j=1}^{n}(1 / n) w_{\rho(j)}}\right. \\
& +(\eta-1) \\
& \left.\times \prod_{j=1}^{n}\left(1-\widetilde{\gamma}_{j}^{L}\right)^{(1 / n) w_{\rho(j)} / \sum_{j=1}^{n}(1 / n) w_{\rho(j)}}\right)^{-1},
\end{aligned}
$$




$$
\begin{aligned}
& \left(\prod_{j=1}^{n}\left(1+(\eta-1) \widetilde{\gamma}_{j}^{U}\right)^{(1 / n) w_{\rho(j)} / \sum_{j=1}^{n}(1 / n) w_{\rho(j)}}\right. \\
& \left.-\prod_{j=1}^{n}\left(1-\widetilde{\gamma}_{j}^{U}\right)^{(1 / n) w_{\rho(j)} / \sum_{j=1}^{n}(1 / n) w_{\rho(j)}}\right) \\
& \times\left(\prod_{j=1}^{n}\left(1+(\eta-1) \widetilde{\gamma}_{j}^{U}\right)^{(1 / n) w_{\rho(j)} / \sum_{j=1}^{n}(1 / n) w_{\rho(j)}}\right. \\
& +(\eta-1) \\
& \times \prod_{j=1}^{n}(1
\end{aligned}
$$

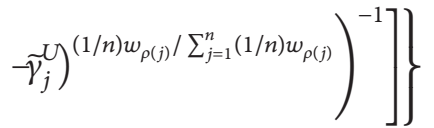

$$
\begin{aligned}
& =\bigcup_{\tilde{\gamma}_{j} \in \tilde{h}_{j}, j=1, \ldots, n}\left\{\left[\left(\prod_{j=1}^{n}\left(1+(\eta-1) \tilde{\gamma}_{j}^{L}\right)^{w_{\rho(j)}}\right.\right.\right. \\
& \left.-\prod_{j=1}^{n}\left(1-\tilde{\gamma}_{j}^{L}\right)^{w_{\rho(j)}}\right) \\
& \times\left(\prod_{j=1}^{n}\left(1+(\eta-1) \tilde{\gamma}_{j}^{L}\right)^{w_{\rho(j)}}\right. \\
& \left.+(\eta-1) \prod_{j=1}^{n}\left(1-\widetilde{\gamma}_{j}^{L}\right)^{w_{\rho(j)}}\right)^{-1}, \\
& \left(\prod_{j=1}^{n}\left(1+(\eta-1) \tilde{\gamma}_{j}^{U}\right)^{w_{\rho(j)}}\right. \\
& \left.-\prod_{j=1}^{n}\left(1-\widetilde{\gamma}_{j}^{U}\right)^{w_{\rho(j)}}\right) \\
& \times\left(\prod_{j=1}^{n}\left(1+(\eta-1) \widetilde{\gamma}_{j}^{U}\right)^{w_{\rho(j)}}\right. \\
& \left.\left.\left.+(\eta-1) \prod_{j=1}^{n}\left(1-\widetilde{\gamma}_{j}^{U}\right)^{w_{\rho(j)}}\right)^{-1}\right]\right\} \\
& =\underset{\text { Hamacher }}{\operatorname{IVHFOWA}}\left(\widetilde{h}_{1}, \widetilde{h}_{2}, \ldots, \widetilde{h}_{n}\right) \text {. }
\end{aligned}
$$

Thus, $\quad$ IVHFSWA $_{\text {Hamacher }}\left(\widetilde{h}_{1}, \widetilde{h}_{2}, \ldots, \widetilde{h}_{n}\right) \quad \stackrel{\omega_{j}=1 / n, j=1, \ldots, n}{\longrightarrow}$ IVHFOWA $_{\text {Hamacher }}\left(\widetilde{h}_{1}, \widetilde{h}_{2}, \ldots, \widetilde{h}_{n}\right)$.

Case 2. If the associated weighting vector $w=$ $\left(w_{1}, w_{2}, \ldots, w_{n}\right)=(1 / n, 1 / n, \ldots, 1 / n)$, then the IVHFHSWA operator is reduced to the IVHFHWA operator.

\section{Proof. Suppose}

$$
\begin{aligned}
& \underset{\text { Hamacher }}{\operatorname{IVHFSWA}}\left(\widetilde{h}_{1}, \widetilde{h}_{2}, \ldots, \widetilde{h}_{n}\right) \\
& =\bigcup_{\tilde{\gamma}_{j} \in \tilde{h}_{j}, j=1, \ldots, n}\left\{\left[\left(\prod_{j=1}^{n}\left(1+(\eta-1) \tilde{\gamma}_{j}^{L}\right)^{\omega_{j}(1 / n) / \sum_{j=1}^{n} \omega_{j}(1 / n)}\right.\right.\right. \\
& \left.-\prod_{j=1}^{n}\left(1-\widetilde{\gamma}_{j}^{L}\right)^{\omega_{j}(1 / n) / \sum_{j=1}^{n} \omega_{j}(1 / n)}\right) \\
& \times\left(\prod_{j=1}^{n}\left(1+(\eta-1) \widetilde{\gamma}_{j}^{L}\right)^{\omega_{j}(1 / n) / \sum_{j=1}^{n} \omega_{j}(1 / n)}\right. \\
& +(\eta-1) \\
& \left.\times \prod_{j=1}^{n}\left(1-\tilde{\gamma}_{j}^{L}\right)^{\omega_{j}(1 / n) / \sum_{j=1}^{n} \omega_{j}(1 / n)}\right)^{-1}, \\
& \left(\prod_{j=1}^{n}\left(1+(\eta-1) \widetilde{\gamma}_{j}^{U}\right)^{\omega_{j}(1 / n) / \sum_{j=1}^{n} \omega_{j}(1 / n)}\right. \\
& \left.-\prod_{j=1}^{n}\left(1-\widetilde{\gamma}_{j}^{U}\right)^{\omega_{j}(1 / n) / \sum_{j=1}^{n} \omega_{j}(1 / n)}\right) \\
& \times\left(\prod_{j=1}^{n}\left(1+(\eta-1) \widetilde{\gamma}_{j}^{U}\right)^{\omega_{j}(1 / n) / \sum_{j=1}^{n} \omega_{j}(1 / n)}\right. \\
& +(\eta-1) \\
& \left.\left.\left.\times \prod_{j=1}^{n}\left(1-\widetilde{\gamma}_{j}^{U}\right)^{\omega_{j}(1 / n) / \sum_{j=1}^{n} \omega_{j}(1 / n)}\right)^{-1}\right]\right\} \\
& =\bigcup_{\widetilde{\gamma}_{j} \in \widetilde{h}_{j}, j=1, \ldots, n}\left\{\left[\left(\prod_{j=1}^{n}\left(1+(\eta-1) \tilde{\gamma}_{j}^{L}\right)^{\omega_{j}}\right.\right.\right. \\
& \left.-\prod_{j=1}^{n}\left(1-\tilde{\gamma}_{j}^{L}\right)^{\omega_{j}}\right) \\
& \times\left(\prod_{j=1}^{n}\left(1+(\eta-1) \tilde{\gamma}_{j}^{L}\right)^{\omega_{j}}\right. \\
& \left.+(\eta-1) \prod_{j=1}^{n}\left(1-\widetilde{\gamma}_{j}^{L}\right)^{\omega_{j}}\right)^{-1}, \\
& \left(\prod_{j=1}^{n}\left(1+(\eta-1) \tilde{\gamma}_{j}^{U}\right)^{\omega_{j}}\right. \\
& \left.-\prod_{j=1}^{n}\left(1-\widetilde{\gamma}_{j}^{U}\right)^{\omega_{j}}\right)
\end{aligned}
$$




$$
\begin{gathered}
\times\left(\prod_{j=1}^{n}\left(1+(\eta-1) \widetilde{\gamma}_{j}^{U}\right)^{\omega_{j}}\right. \\
\left.\left.\left.+(\eta-1) \prod_{j=1}^{n}\left(1-\widetilde{\gamma}_{j}^{U}\right)^{\omega_{j}}\right)^{-1}\right]\right\} \\
=\underset{\text { Hamacher }}{\operatorname{IVHFWA}}\left(\widetilde{h}_{1}, \widetilde{h}_{2}, \ldots, \widetilde{h}_{n}\right) .
\end{gathered}
$$

Thus, $\quad$ IVHFSWA $_{\text {Hamacher }}\left(\widetilde{h}_{1}, \widetilde{h}_{2}, \ldots, \widetilde{h}_{n}\right) \quad \stackrel{w_{j}=1 / n, j=1, \ldots, n}{\longrightarrow}$ IVHFWA $_{\text {Hamacher }}\left(\widetilde{h}_{1}, \widetilde{h}_{2}, \ldots, \widetilde{h}_{n}\right)$.

Case 3. If $w=\left(w_{1}, w_{2}, \ldots, w_{n}\right)=(1 / n, 1 / n, \ldots, 1 / n)$ and $\omega=\left(\omega_{1}, \omega_{2}, \ldots, \omega_{n}\right)=(1 / n, 1 / n, \ldots, 1 / n)$, then the IVHFHSWA operator results in an interval-valued hesitant fuzzy Hamacher averaging (IVHFHA) operator:

$$
\begin{aligned}
& \underset{\text { Hamacher }}{\operatorname{IVHFA}}\left(\widetilde{h}_{1}, \widetilde{h}_{2}, \ldots, \widetilde{h}_{n}\right) \\
& =\bigoplus_{j=1}^{n} \frac{1}{n} \widetilde{h}_{j} \\
& =\bigcup_{\tilde{\gamma}_{j} \in \tilde{h}_{j}, j=1, \ldots, n}\left\{\left[\left(\prod_{j=1}^{n}\left(1+(\eta-1) \tilde{\gamma}_{j}^{L}\right)^{1 / n}\right.\right.\right. \\
& \left.-\prod_{j=1}^{n}\left(1-\widetilde{\gamma}_{j}^{L}\right)^{1 / n}\right) \\
& \times\left(\prod_{j=1}^{n}\left(1+(\eta-1) \tilde{\gamma}_{j}^{L}\right)^{1 / n}\right. \\
& \left.+(\eta-1) \prod_{j=1}^{n}\left(1-\tilde{\gamma}_{j}^{L}\right)^{1 / n}\right)^{-1}, \\
& \left(\prod_{j=1}^{n}\left(1+(\eta-1) \widetilde{\gamma}_{j}^{U}\right)^{1 / n}\right. \\
& \left.-\prod_{j=1}^{n}\left(1-\widetilde{\gamma}_{j}^{U}\right)^{1 / n}\right) \\
& \times\left(\prod_{j=1}^{n}\left(1+(\eta-1) \widetilde{\gamma}_{j}^{U}\right)^{1 / n}\right. \\
& \left.\left.\left.+(\eta-1) \prod_{j=1}^{n}\left(1-\widetilde{\gamma}_{j}^{U}\right)^{1 / n}\right)^{-1}\right]\right\} .
\end{aligned}
$$

From Examples 12, 16, and 18 as well as Cases 1 and 2, it follows that the IVHFHSWA operator generalizes both the IVHFHWA operator and the IVHFHOWA operator, and thus it can reflect the importance of both the considered argument and its ordered position.

\subsection{Interval-Valued Hesitant Fuzzy Hamacher Synergetic Weighted Geometric Operator}

Definition 22. Let $\widetilde{h}_{j}=\bigcup_{\widetilde{\gamma}_{j} \tilde{h}_{j}}\left\{\widetilde{\gamma}_{j}=\left[\tilde{\gamma}_{j}^{L}, \widetilde{\gamma}_{j}^{U}\right]\right\}(j=1,2, \ldots, n)$ be a collection of IVHFEs, and $\omega=\left(\omega_{1}, \omega_{2}, \ldots, \omega_{n}\right)^{T}$ is the relative weighting vector of the $\widetilde{h}_{j}(i=1,2, \ldots, n)$, with $\omega_{i} \in[0,1]$ and $\sum_{i=1}^{n} \omega_{i}=1$. Then an interval-valued hesitant fuzzy Hamacher synergetic weighted geometric (IVHFHSWG) operator is a mapping IVHFSWG Hamacher $: \widetilde{H}^{n} \rightarrow \widetilde{H}$, associated with a weighting vector $w=\left(w_{1}, w_{2}, \ldots, w_{n}\right)$, such that $w_{i} \in[0,1]$ and $\sum_{i=1}^{n} w_{i}=1$, according to the following expression:

$$
\begin{aligned}
& \underset{\text { Hamacher }}{\operatorname{IVHFSWG}}\left(\widetilde{h}_{1}, \widetilde{h}_{2}, \ldots, \widetilde{h}_{n}\right) \\
& =\bigotimes_{j=1}^{n} \widetilde{h}_{j}^{\omega_{j} w_{\rho(j)} / \sum_{j=1}^{n} \omega_{j} w_{\rho(j)}} \\
& =\bigcup_{\tilde{\gamma}_{j} \in \tilde{h}_{j}, j=1, \ldots, n}\left\{\left[\left(\eta \prod_{j=1}^{n}\left(\tilde{\gamma}_{j}^{L}\right)^{\omega_{j} w_{\rho(j)} / \sum_{j=1}^{n} \omega_{j} w_{\rho(j)}}\right)\right.\right. \\
& \times\left(\prod_{j=1}^{n}(1+(\eta-1)\right. \\
& \left.\times\left(1-\tilde{\gamma}_{j}^{L}\right)\right)^{\omega_{j} w_{\rho(j)} / \sum_{j=1}^{n} \omega_{j} w_{\rho(j)}} \\
& +(\eta-1) \\
& \left.\times \prod_{j=1}^{n}\left(\widetilde{\gamma}_{j}^{L}\right)^{\omega_{j} w_{\rho(j)} / \sum_{j=1}^{n} \omega_{j} w_{\rho(j)}}\right)^{-1}, \\
& \left(\eta \prod_{j=1}^{n}\left(\widetilde{\gamma}_{j}^{U}\right)^{\omega_{j} w_{\rho(j)} / \sum_{j=1}^{n} \omega_{j} w_{\rho(j)}}\right) \\
& \times\left(\prod_{j=1}^{n}(1+(\eta-1)\right. \\
& \left.\times\left(1-\widetilde{\gamma}_{j}^{U}\right)\right)^{\omega_{j} \omega_{\rho(j)} / \sum_{j=1}^{n} \omega_{j} \omega_{\rho(j)}} \\
& +(\eta-1) \\
& \left.\left.\left.\times \prod_{j=1}^{n}\left(\tilde{\gamma}_{j}^{U}\right)^{\omega_{j} w_{\rho(j)} / \sum_{j=1}^{n} \omega_{j} \omega_{\rho(j)}}\right)^{-1}\right]\right\},
\end{aligned}
$$

where $\rho:\{1,2, \ldots, n\} \rightarrow\{1,2, \ldots, n\}$ is a permutation function such that $\widetilde{h}_{j}$ is the $\rho(j)$ th largest element of the collection of $\widetilde{h}_{j}(i=1,2, \ldots, n)$. In particular, if all $\widetilde{\gamma}_{j}^{L}=\widetilde{\gamma}_{j}^{U}$, 
the IVHFEs $\tilde{h}_{j}=\bigcup_{\tilde{\gamma}_{j} \in \tilde{h}_{j}}\left\{\tilde{\gamma}_{j}=\left[\tilde{\gamma}_{j}^{L}, \widetilde{\gamma}_{j}^{U}\right]\right\}(j=1,2, \ldots, n)$ are reduced to HFEs $h_{j}=\bigcup_{\gamma_{j} \in h_{j}}\left\{\gamma_{j}\right\}(j=1,2, \ldots, n)$; then the IVHFHSWG operator becomes a hesitant fuzzy Hamacher synergetic weighted geometric (HFHSWG) operator:

$$
\begin{aligned}
& \underset{\text { Hamacher }}{\operatorname{HFSWG}}\left(\tilde{h}_{1}, \tilde{h}_{2}, \ldots, \tilde{h}_{n}\right) \\
& =\bigotimes_{\gamma_{j} \in h_{j}, j=1, \ldots, n}^{n} h_{j}^{\omega_{j} w_{\rho(j)} / \sum_{j=1}^{n} \omega_{j} w_{\rho(j)}}\left\{\begin{array}{c}
\left.\eta \prod_{j=1}^{n}\left(\gamma_{j}\right)^{\left.\omega_{j} w_{\rho(j)} / \sum_{j=1}^{n} \omega_{j} w_{\rho(j)}\right)}\right) \\
\times\left(\prod_{j=1}^{n}(1+(\eta-1)\right. \\
\quad+(\eta-1) \\
\left.\times \prod_{j=1}^{n}\left(\gamma_{j}\right)^{\omega_{j} w_{\rho(j)} / \sum_{j=1}^{n} \omega_{j} w_{\rho(j)}}\right)^{-1}
\end{array} .\right.
\end{aligned}
$$

Example 23. Given the collection of IVHFEs, $\tilde{h}_{1}=$ $\{[0.2,0.4],[0.5,0.7],[0.6,0.8]\}$ and $\widetilde{h}_{2}=\{[0.4,0.5],[0.7,0.8]\}$, the relative weights $\omega_{1}=0.3, \omega_{2}=0.7$ and the associated weight vectors are $w_{1}=0.6, w_{2}=0.4$, and suppose that the $\eta=2$, then, since $\widetilde{h}_{1}<\widetilde{h}_{2}$, and the associated weights of $\widetilde{h}_{1}$ and $\widetilde{h}_{2}$ are $w_{\rho(1)}=0.4$ and $w_{\rho(2)}=0.6$, respectively, then

$$
\begin{aligned}
& \underset{\text { Hamacher }}{\operatorname{IVHFSWG}}\left(\widetilde{h}_{1}, \widetilde{h}_{2}\right) \\
& =\bigotimes_{j=1}^{2} \tilde{h}_{j}^{w_{\rho(j)} \omega_{j} / \sum_{j=1}^{2} w_{\rho(j)} \omega_{j}} \\
& =\bigcup_{\tilde{\gamma}_{j} \in \tilde{h}_{j}, j=1,2}\left\{\left[\left(2 \prod_{j=1}^{2}\left(\tilde{\gamma}_{j}^{L}\right)^{\omega_{j} w_{\rho(j)} / \sum_{j=1}^{n} \omega_{j} w_{\rho(j)}}\right)\right.\right. \\
& \times\left(\prod_{j=1}^{2}\left(2-\widetilde{\gamma}_{j}^{L}\right)^{\omega_{j} w_{\rho(j)} / \sum_{j=1}^{n} \omega_{j} w_{\rho(j)}}\right. \\
& \left.+\prod_{j=1}^{2}\left(\widetilde{\gamma}_{j}^{L}\right)^{\omega_{j} w_{\rho(j)} / \sum_{j=1}^{n} \omega_{j} w_{\rho(j)}}\right)^{-1}, \\
& \left(2 \prod_{j=1}^{2}\left(\widetilde{\gamma}_{j}^{U}\right)^{\omega_{j} w_{\rho(j)} / \sum_{j=1}^{n} \omega_{j} w_{\rho(j)}}\right) \\
& \times\left(\prod_{j=1}^{2}\left(2-\widetilde{\gamma}_{j}^{U}\right)^{\omega_{j} w_{\rho(j)} / \sum_{j=1}^{n} \omega_{j} w_{\rho(j)}}\right. \\
& \left.\left.\left.+\prod_{j=1}^{2}\left(\widetilde{\gamma}_{j}^{U}\right)^{\omega_{j} w_{\rho(j)} / \sum_{j=1}^{n} \omega_{j} w_{\rho(j)}}\right)^{-1}\right]\right\}
\end{aligned}
$$

$$
\begin{array}{r}
=\{[0.346,0.477],[0.551,0.699],[0.421,0.541], \\
[0.653,0.778],[0.439,0.559],[0.677,0.8]\} .
\end{array}
$$

Furthermore, we can obtain the aggregated results corresponding to some special cases of the parameter $\eta$, which are shown in Table 1.

From Table 1, we know that the aggregated results derived from the IVHFHSWG steadily increases as the parameter $\eta$ increases, which implies that the IVHFHSWG operator with parameters can provide the decision makers more choices and thus the aggregated results are more flexible than the existing ones, because we can choose different values of the parameter according to the different situations.

The IVHFHSWG operator has some essential properties, such as idempotency, boundary, and monotonicity.

Theorem 24 (Idempotency). Let $\widetilde{h}_{j}=\bigcup_{\tilde{\gamma}_{j} \in \tilde{h}_{j}}\left\{\tilde{\gamma}_{j}\right\}(j=$ $1,2, \ldots, n)$ be a collection of IVHFEs. If $\widetilde{h}_{j}=\widetilde{h}=\bigcup_{\tilde{\gamma} \in \tilde{h}}\{\tilde{\gamma}=$ $\left.\left[\widetilde{\gamma}^{L}, \widetilde{\gamma}^{U}\right]\right\}$ for all $j=1,2, \ldots, n$, then

$$
\underset{\text { Hamacher }}{\operatorname{IVHFSWG}}\left(\tilde{h}_{1}, \tilde{h}_{2}, \ldots, \tilde{h}_{n}\right)=\tilde{h} .
$$

Theorem 25 (Boundedness). Let $\tilde{h}_{j}=\bigcup_{\tilde{\gamma}_{j} \in \tilde{h}_{j}}\left\{\tilde{\gamma}_{j}=\right.$ $\left.\left[\widetilde{\gamma}_{j}^{L}, \widetilde{\gamma}_{j}^{U}\right]\right\}(j=1,2, \ldots, n)$ be a collection of IVHFEs, $\bigcap_{j=1}^{n} \widetilde{h}_{j}=$ $\bigcup_{\tilde{\gamma}_{j} \in \tilde{h}_{j}, j=1,2, \ldots, n}\left\{\left[\min _{1 \leq j \leq n}\left\{\widetilde{\gamma}_{j}^{L}\right\}, \min _{1 \leq j \leq n}\left\{\widetilde{\gamma}_{j}^{U}\right\}\right]\right\}$, and $\bigcup_{j=1}^{n} \widetilde{h}_{j}=$ $\bigcup_{\tilde{\gamma}_{j} \in \tilde{h}_{j}, j=1,2, \ldots, n}\left\{\left[\max _{1 \leq j \leq n}\left\{\tilde{\gamma}_{j}^{L}\right\}, \max _{1 \leq j \leq n}\left\{\tilde{\gamma}_{j}^{U}\right\}\right]\right\}$, then

$$
\bigcap_{j=1}^{n} \tilde{h}_{j} \leq \underset{\text { Hamacher }}{\operatorname{IVHFWG}}\left(\widetilde{h}_{1}, \widetilde{h}_{2}, \ldots, \widetilde{h}_{n}\right) \leq \bigcup_{j=1}^{n} \widetilde{h}_{j} .
$$

Theorem 26 (Monotonicity). Let $\widetilde{h}_{j}=\bigcup_{\widetilde{\gamma}_{j} \in \tilde{h}_{j}}\left\{\widetilde{\gamma}_{j}\right\}(j=$ $1,2, \ldots, n)$ be a collection of IVHFEs. If $h_{j} \leq h_{j}^{\prime}$ for all $j=$ $1,2, \ldots, n$, then

$$
\operatorname{IVHFSWG}\left(\widetilde{h}_{1}, \tilde{h}_{2}, \ldots, \tilde{h}_{n}\right) \leq \underset{\text { Hamacher }}{\operatorname{IVHFSWG}}\left(\widetilde{h}_{1}^{\prime}, \widetilde{h}_{2}^{\prime}, \ldots, \widetilde{h}_{n}^{\prime}\right) .
$$

In the following, we discuss the special cases of the IVHFHSWG operator.

(1) From the perspective of parameter $\eta$.

Case 1. If $\eta=1$, then the IVHFHSWG operator results in an interval-valued hesitant fuzzy synergetic weighted geometric (IVHFSWG) operator:

$$
\begin{aligned}
\operatorname{IVHFSWG}\left(\tilde{h}_{1}, \tilde{h}_{2}, \ldots, \tilde{h}_{n}\right) \\
=\bigcup_{\tilde{\gamma}_{j} \in \tilde{h}_{j}, j=1, \ldots, n}\left\{\left[\prod_{j=1}^{n}\left(\widetilde{\gamma}_{j}^{L}\right)^{\omega_{j} w_{\rho(j)} / \sum_{j=1}^{n} \omega_{j} w_{\rho(j)}},\right.\right. \\
\\
\left.\left.\prod_{j=1}^{n}\left(\widetilde{\gamma}_{j}^{U}\right)^{\omega_{j} w_{\rho(j)} / \sum_{j=1}^{n} \omega_{j} w_{\rho(j)}}\right]\right\} .
\end{aligned}
$$


TABLE 1: Aggregated results for the different $\eta$.

\begin{tabular}{lccc}
\hline ID & $\eta$ & Aggregated results & Score values \\
\hline 1 & 0.1 & $\{[0.332,0.474],[0.470,0.661],[0.419,0.534],[0.644,0.776],[0.433,0.546],[0.675,0.8]\}$ & {$[0.496,0.632]$} \\
2 & 0.5 & $\{[0.340,0.475],[0.509,0.676],[0.420,0.537],[0.648,0.776],[0.435,0.551],[0.676,0.8]\}$ & {$[0.505,0.636]$} \\
3 & 1 & $\{[0.343,0.476],[0.531,0.687],[0.420,0.538],[0.650,0.777],[0.437,0.554],[0.677,0.8]\}$ & {$[0.510,0.639]$} \\
4 & 1.5 & $\{[0.345,0.476],[0.543,0.694],[0.420,0.540],[0.652,0.777],[0.438,0.557],[0.677,0.8]\}$ & {$[0.513,0.641]$} \\
5 & 2 & $\{[0.346,0.477],[0.551,0.699],[0.421,0.541],[0.653,0.778],[0.439,0.559],[0.677,0.8]\}$ & {$[0.515,0.642]$} \\
6 & 5 & $\{[0.348,0.477],[0.570,0.713],[0.421,0.543],[0.656,0.779],[0.441,0.566],[0.678,0.8]\}$ & {$[0.519,0.646]$} \\
7 & 100 & $\{[0.349,0.478],[0.587,0.728],[0.422,0.546],[0.659,0.780],[0.443,0.575],[0.679,0.8]\}$ & {$[0.523,0.651]$} \\
\hline
\end{tabular}

Case 2. If $\eta=2$, then the IVHFHSWG operator results in an interval-valued hesitant fuzzy Einstein synergetic weighted geometric (IVHFESWG) operator:

$$
\begin{aligned}
& \operatorname{IVHFESWG}\left(\tilde{h}_{1}, \tilde{h}_{2}, \ldots, \tilde{h}_{n}\right) \\
& =\bigcup_{\tilde{\gamma}_{j} \in \widetilde{h}_{j}, j=1, \ldots, n}\left\{\left[\left(2 \prod_{j=1}^{n}\left(\widetilde{\gamma}_{j}^{L}\right)^{\omega_{j} w_{\rho \lambda(j)} / \sum_{j=1}^{n} \omega_{j} w_{\rho \lambda(j)}}\right)\right.\right. \\
& \times\left(\prod_{j=1}^{n}\left(2-\widetilde{\gamma}_{j}^{L}\right)^{\omega_{j} w_{\rho \lambda(j)} / \sum_{j=1}^{n} \omega_{j} w_{\rho \lambda(j)}}\right. \\
& \left.+\prod_{j=1}^{n}\left(\widetilde{\gamma}_{j}^{L}\right)^{\omega_{j} w_{\rho \lambda(j)} / \sum_{j=1}^{n} \omega_{j} w_{\rho \lambda(j)}}\right)^{-1}, \\
& \left(2 \prod_{j=1}^{n}\left(\tilde{\gamma}_{j}^{U}\right)^{\omega_{j} w_{\rho \lambda(j)} / \sum_{j=1}^{n} \omega_{j} w_{\rho \lambda(j)}}\right) \\
& \times\left(\prod_{j=1}^{n}\left(2-\widetilde{\gamma}_{j}^{U}\right)^{\omega_{j} w_{\rho \lambda(j)} / \sum_{j=1}^{n} \omega_{j} w_{\rho \lambda(j)}}\right. \\
& \left.\left.\left.+\prod_{j=1}^{n}\left(\widetilde{\gamma}_{j}^{U}\right)^{\omega_{j} w_{\rho \lambda(j)} / \sum_{j=1}^{n} \omega_{j} w_{\rho \lambda(j)}}\right)^{-1}\right]\right\} .
\end{aligned}
$$

(2) From the perspective of the types of weights.

Case 1. If the relative weighting vector $\omega=\left(\omega_{1}, \omega_{2}, \ldots, \omega_{n}\right)=$ $(1 / n, 1 / n, \ldots, 1 / n)$, then the IVHFHSWG operator becomes an interval-valued hesitant fuzzy Hamacher ordered weighted geometric (IVHFHOWG) operator:

$$
\begin{aligned}
& \underset{\text { Hamacher }}{\operatorname{IVHFSWG}}\left(\widetilde{h}_{1}, \widetilde{h}_{2}, \ldots, \widetilde{h}_{n}\right) \\
& \stackrel{\omega_{j}=1 / n, j=1, \ldots, n}{\longrightarrow} \operatorname{IVHFOWWG}\left(\widetilde{h}_{1}, \widetilde{h}_{2}, \ldots, \widetilde{h}_{n}\right) \\
& =\bigotimes_{j=1}^{n} \tilde{h}_{j}^{w_{\rho(j)}}
\end{aligned}
$$

$$
\begin{aligned}
=\bigcup_{\tilde{\gamma}_{j} \in \tilde{h}_{j}, j=1, \ldots, n}\{ & \left(\eta \prod_{j=1}^{n}\left(\tilde{\gamma}_{j}^{L}\right)^{w_{\rho(j)}}\right) \\
& \times\left(\prod_{j=1}^{n}\left(1+(\eta-1)\left(1-\tilde{\gamma}_{j}^{L}\right)\right)^{w_{\rho(j)}}\right. \\
& \left.+(\eta-1) \prod_{j=1}^{n}\left(\tilde{\gamma}_{j}^{L}\right)^{w_{\rho(j)}}\right)^{-1}, \\
& \left(\eta \prod_{j=1}^{n}\left(\tilde{\gamma}_{j}^{U}\right)^{w_{\rho(j)}}\right) \\
& \times\left(\prod_{j=1}^{n}\left(1+(\eta-1)\left(1-\widetilde{\gamma}_{j}^{U}\right)\right)^{w_{\rho(j)}}\right. \\
& \left.\left.\left.+(\eta-1) \prod_{j=1}^{n}\left(\tilde{\gamma}_{j}^{U}\right)^{w_{\rho(j)}}\right)^{-1}\right]\right\} .
\end{aligned}
$$

On the other hand

$$
\begin{aligned}
& \underset{\text { Hamacher }}{\operatorname{IVHFSWG}}\left(\widetilde{h}_{1}, \tilde{h}_{2}, \ldots, \tilde{h}_{n}\right) \\
& \stackrel{\omega_{j}=1 / n, j=1, \ldots, n}{\longrightarrow} \operatorname{IVHFOWG}\left(\widetilde{h}_{1}, \widetilde{h}_{2}, \ldots, \widetilde{h}_{n}\right) \\
& =\bigotimes_{j=1}^{n} \tilde{h}_{\sigma(j)}^{w_{j}} \\
& =\bigcup_{\tilde{\gamma}_{j} \in \widetilde{h}_{j}, j=1, \ldots, n}\left\{\left[\left(\eta \prod_{j=1}^{n}\left(\tilde{\gamma}_{\sigma(j)}^{L}\right)\right)^{w_{j}}\right)\right. \\
& \quad \times\left(\prod_{j=1}^{n}\left(1+(\eta-1)\left(1-\widetilde{\gamma}_{\sigma(j)}^{L}\right)\right)^{w_{j}}\right.
\end{aligned}
$$




$$
\begin{aligned}
& \left(\eta \prod_{j=1}^{n}\left(\widetilde{\gamma}_{\sigma(j)}^{U}\right)^{w_{j}}\right) \\
& \times\left(\prod_{j=1}^{n}\left(1+(\eta-1)\left(1-\widetilde{\gamma}_{\sigma(j)}^{U}\right)\right)^{w_{j}}\right. \\
& \left.\left.\left.\quad+(\eta-1) \prod_{j=1}^{n}\left(\widetilde{\gamma}_{\sigma(j)}^{U}\right)^{w_{j}}\right)^{-1}\right]\right\} .
\end{aligned}
$$

Furthermore, similar to Theorems 10 and 11, the following theorems hold.

Theorem 27. The IVHFOWG operator proposed by Chen et al. [11] is the special case of the IVHFHOWG operator; that is, if $\eta=1$, then

$$
\begin{aligned}
& \underset{\text { Hamacher }}{\operatorname{IVHFOWG}}\left(\widetilde{h}_{1}, \widetilde{h}_{2}, \ldots, \widetilde{h}_{n}\right) \stackrel{\eta=1}{\longrightarrow} \operatorname{IVHFOWG}\left(\widetilde{h}_{1}, \widetilde{h}_{2}, \ldots, \widetilde{h}_{n}\right) \\
& =\bigcup_{\tilde{\gamma}_{j} \in \widetilde{h}_{j}, j=1, \ldots, n}\left\{\left[\prod_{j=1}^{n}\left(\tilde{\gamma}_{\sigma(j)}^{L}\right)^{w_{j}}, \prod_{j=1}^{n}\left(\widetilde{\gamma}_{\sigma(j)}^{U}\right)^{w_{j}}\right]\right\} .
\end{aligned}
$$

On the other hand

$$
\begin{aligned}
& \underset{\text { Hamacher }}{\operatorname{VHFOWG}}\left(\widetilde{h}_{1}, \widetilde{h}_{2}, \ldots, \widetilde{h}_{n}\right) \stackrel{\eta=1}{\longrightarrow} \operatorname{IVHFOWG}\left(\widetilde{h}_{1}, \widetilde{h}_{2}, \ldots, \widetilde{h}_{n}\right) \\
& =\bigcup_{\tilde{\gamma}_{j} \in \tilde{h}_{j}, j=1, \ldots, n}\left\{\left[\prod_{j=1}^{n}\left(\tilde{\gamma}_{j}^{L}\right)^{w_{\rho(j)}}, \prod_{j=1}^{n}\left(\widetilde{\gamma}_{j}^{U}\right)^{w_{\rho(j)}}\right]\right\} .
\end{aligned}
$$

Theorem 28. The IVHFEOWG operator proposed by Wei and Zhao [23] is the special case of the IVHFHOWG operator; that is, if $\eta=2$, then

$$
\begin{aligned}
& \underset{\text { Hamacher }}{\operatorname{IVHFOWG}}\left(\widetilde{h}_{1}, \widetilde{h}_{2}, \ldots, \widetilde{h}_{n}\right) \\
& \stackrel{\eta=2}{\longrightarrow} \operatorname{IVHFEOWG}\left(\widetilde{h}_{1}, \widetilde{h}_{2}, \ldots, \widetilde{h}_{n}\right) \\
& =\bigcup_{\tilde{\gamma}_{j} \in \tilde{h}_{j}, j=1, \ldots, n}\left\{\left[\left(2 \prod_{j=1}^{n}\left(\tilde{\gamma}_{\sigma(j)}^{L}\right)^{w_{j}}\right)\right.\right. \\
& \times\left(\prod_{j=1}^{n}\left(1+\left(1-\widetilde{\gamma}_{\sigma(j)}^{L}\right)\right)^{w_{j}}\right. \\
& \left.+\prod_{j=1}^{n}\left(\widetilde{\gamma}_{\sigma(j)}^{L}\right)^{w_{j}}\right)^{-1}
\end{aligned}
$$

$$
\begin{gathered}
\left(2 \prod_{j=1}^{n}\left(\widetilde{\gamma}_{\sigma(j)}^{U}\right)^{w_{j}}\right) \\
\times\left(\prod_{j=1}^{n}\left(1+\left(1-\widetilde{\gamma}_{\sigma(j)}^{U}\right)\right)^{w_{j}}\right. \\
\left.\left.\left.\quad+\prod_{j=1}^{n}\left(\widetilde{\gamma}_{\sigma(j)}^{U}\right)^{w_{j}}\right)^{-1}\right]\right\} .
\end{gathered}
$$

On the other hand

$$
\begin{aligned}
& \underset{\text { Hamacher }}{\operatorname{VHFOWG}}\left(\widetilde{h}_{1}, \widetilde{h}_{2}, \ldots, \widetilde{h}_{n}\right) \\
& \stackrel{\eta=2}{\longrightarrow} \operatorname{IVHFEOWG}\left(\widetilde{h}_{1}, \widetilde{h}_{2}, \ldots, \widetilde{h}_{n}\right) \\
& =\bigcup_{\widetilde{\gamma}_{j} \in \widetilde{h}_{j}, j=1, \ldots, n}\left\{\left[\left(2 \prod_{j=1}^{n}\left(\widetilde{\gamma}_{j}^{L}\right)^{w_{\rho(j)}}\right)\right.\right. \\
& \times\left(\prod_{j=1}^{n}\left(1+\left(1-\tilde{\gamma}_{j}^{L}\right)\right)^{w_{\rho(j)}}\right. \\
& \left.+\prod_{j=1}^{n}\left(\tilde{\gamma}_{j}^{L}\right)^{w_{\rho(j)}}\right)^{-1}, \\
& \left(2 \prod_{j=1}^{n}\left(\widetilde{\gamma}_{j}^{U}\right)^{w_{\rho(j)}}\right) \\
& \times\left(\prod_{j=1}^{n}\left(1+\left(1-\widetilde{\gamma}_{j}^{U}\right)\right)^{w_{\rho(j)}}\right. \\
& \left.\left.\left.+\prod_{j=1}^{n}\left(\widetilde{\gamma}_{j}^{U}\right)^{w_{\rho(j)}}\right)^{-1}\right]\right\} .
\end{aligned}
$$

Case 2. If the associated weighting vector $w=\left(w_{1}, w_{2}\right.$, $\left.\ldots, w_{n}\right)=(1 / n, 1 / n, \ldots, 1 / n)$, then the IVHFHSWG operator becomes an interval-valued hesitant fuzzy Hamacher weighted geometric (IVHFHWG) operator:

$$
\begin{aligned}
& \underset{\text { Hamacher }}{\operatorname{IVHFSWG}}\left(\widetilde{h}_{1}, \widetilde{h}_{2}, \ldots, \widetilde{h}_{n}\right) \\
& \quad \stackrel{w_{j}=1 / n, j=1, \ldots, n}{\longrightarrow} \underset{\text { Hamacher }}{\operatorname{IVHFWG}}\left(\widetilde{h}_{1}, \widetilde{h}_{2}, \ldots, \widetilde{h}_{n}\right) \\
& =\bigotimes_{j=1}^{n} \widetilde{h}_{j}^{\omega_{j}}
\end{aligned}
$$




$$
\begin{aligned}
=\bigcup_{\tilde{\gamma}_{j} \in \tilde{h}_{j}, j=1, \ldots, n}\{ & \left(\eta \prod_{j=1}^{n}\left(\tilde{\gamma}_{j}^{L}\right)^{\omega_{j}}\right) \\
& \times\left(\prod_{j=1}^{n}\left(1+(\eta-1)\left(1-\tilde{\gamma}_{j}^{L}\right)\right)^{\omega_{j}}\right. \\
& +\left(\eta \prod_{j=1}^{n}\left(\tilde{\gamma}_{j}^{U}\right)^{\omega_{j}}\right) \\
& \times\left(\prod_{j=1}^{n}\left(1+(\eta-1)\left(1-\tilde{\gamma}_{j}^{U}\right)\right)^{\omega_{j}}\left(\tilde{\gamma}_{j}^{L}\right)^{\omega_{j}}\right)^{-1}, \\
& \\
& \\
& \left.\left.\left.+(\eta-1) \prod_{j=1}^{n}\left(\widetilde{\gamma}_{j}^{U}\right)^{\omega_{j}}\right)^{-1}\right]\right\} .
\end{aligned}
$$

Furthermore, the following theorems hold.

Theorem 29. The IVHFWG operator proposed by Chen et al. [11] is the special case of the IVHFHWG operator; that is, if $\eta=1$, then

$$
\begin{aligned}
& \underset{\text { Hamacher }}{\operatorname{IVHFWG}}\left(\widetilde{h}_{1}, \widetilde{h}_{2}, \ldots, \widetilde{h}_{n}\right) \stackrel{\eta=1}{\longrightarrow} \operatorname{IVHFWG}\left(\widetilde{h}_{1}, \widetilde{h}_{2}, \ldots, \widetilde{h}_{n}\right) \\
& =\bigcup_{\tilde{\gamma}_{j} \in \widetilde{h}_{j}, j=1, \ldots, n}\left\{\left[\prod_{j=1}^{n}\left(\tilde{\gamma}_{j}^{L}\right)^{\omega_{j}}, \prod_{j=1}^{n}\left(\widetilde{\gamma}_{j}^{U}\right)^{\omega_{j}}\right]\right\} .
\end{aligned}
$$

Theorem 30. The IVHFEWG operator proposed by Wei and Zhao [23] is the special case of the IVHFHWG operator; that is, if $\eta=2$, then

$$
\begin{array}{r}
\underset{\text { Hamacher }}{\operatorname{IVHFWG}}\left(\widetilde{h}_{1}, \widetilde{h}_{2}, \ldots, \widetilde{h}_{n}\right) \stackrel{\eta=2}{\longrightarrow} \operatorname{IVHFEWG}\left(\widetilde{h}_{1}, \widetilde{h}_{2}, \ldots, \widetilde{h}_{n}\right) \\
=\bigcup_{\tilde{\gamma}_{j} \in \tilde{h}_{j}, j=1, \ldots, n}\left\{\left[\frac{2 \prod_{j=1}^{n}\left(\tilde{\gamma}_{j}^{L}\right)^{\omega_{j}}}{\prod_{j=1}^{n}\left(1+\left(1-\tilde{\gamma}_{j}^{L}\right)\right)^{\omega_{j}}+\prod_{j=1}^{n}\left(\tilde{\gamma}_{j}^{L}\right)^{\omega_{j}}},\right.\right. \\
\\
\left.\left.\frac{2 \prod_{j=1}^{n}\left(\tilde{\gamma}_{j}^{U}\right)^{\omega_{j}}}{\prod_{j=1}^{n}\left(1+\left(1-\tilde{\gamma}_{j}^{U}\right)\right)^{\omega_{j}}+\prod_{j=1}^{n}\left(\tilde{\gamma}_{j}^{U}\right)^{\omega_{j}}}\right]\right\} .
\end{array}
$$

Case 3. If $w=\left(w_{1}, w_{2}, \ldots, w_{n}\right)=(1 / n, 1 / n, \ldots, 1 / n)$ and $\omega=\left(\omega_{1}, \omega_{2}, \ldots, \omega_{n}\right)=(1 / n, 1 / n, \ldots, 1 / n)$, then the IVHFHSWG operator becomes an interval-valued hesitant fuzzy Hamacher geometric (IVHFHG) operator:

$$
\begin{aligned}
& \underset{\text { Hamacher }}{\operatorname{IVHFSWG}}\left(\widetilde{h}_{1}, \widetilde{h}_{2}, \ldots, \widetilde{h}_{n}\right) \\
& \underset{w_{j}=1 / n, j=1, \ldots, n}{\stackrel{\omega_{j}=1 / n, j=1, \ldots, n}{\longrightarrow}} \text { HVHFG }\left(\tilde{h}_{1}, \tilde{h}_{2}, \ldots, \tilde{h}_{n}\right) \\
& =\underset{\tilde{\gamma}_{j} \in \tilde{h}_{j}, j=1, \ldots, n}{\bigcup}\left\{\left[\left(\eta \prod_{j=1}^{n}\left(\tilde{\gamma}_{j}^{L}\right)^{1 / n}\right)\right.\right. \\
& \times\left(\prod_{j=1}^{n}\left(1+(\eta-1)\left(1-\widetilde{\gamma}_{j}^{L}\right)\right)^{1 / n}\right. \\
& \left.+(\eta-1) \prod_{j=1}^{n}\left(\tilde{\gamma}_{j}^{L}\right)^{1 / n}\right)^{-1}, \\
& \left(\eta \prod_{j=1}^{n}\left(\widetilde{\gamma}_{j}^{U}\right)^{1 / n}\right) \\
& \times\left(\prod_{j=1}^{n}\left(1+(\eta-1)\left(1-\widetilde{\gamma}_{j}^{U}\right)\right)^{1 / n}\right. \\
& \left.\left.\left.+(\eta-1) \prod_{j=1}^{n}\left(\widetilde{\gamma}_{j}^{U}\right)^{1 / n}\right)^{-1}\right]\right\} .
\end{aligned}
$$

When applying the IVHFHSW (IVHFHSWA and IVHFHSWG) operators for decision making, the key issue is to determine the associated weights. Different approaches have been suggested for obtaining the associated weight vector $[31,32]$. The most common of them is the one based on the use of a linguistic quantifier [4, 32, 33], Q, which is a regular increasing monotonic (RIM) function $Q:[0,1] \rightarrow[0,1]$ that satisfies $Q(0)=0, Q(1)=1$, and $Q(x) \geq Q(y)$ if $x>y$, where $Q$ is substituted with a linguistic quantifier. In the quantifier-guided aggregation process, the decision makers provide a decision strategy with a linguistic quantifier that indicates the portion of the criteria they feel is necessary for a good solution, such as "many" or "most." The formal decision strategy is that " $Q$ criteria must be satisfied by an acceptable alternative." Yager [32] recommended obtaining the descending orders associated weights based on a linguistic quantifier as follows:

$$
w_{i}=Q\left(\frac{i}{n}\right)-Q\left(\frac{i-1}{n}\right), \quad i=1, \ldots, n
$$

where $Q$ is a linguistic quantifier and the simplest and most common one is defined as

$$
Q(r)=r^{\alpha}, \quad \alpha \geq 0, r \in[0,1]
$$


TABLE 2: Guideline to select linguistic quantifiers and equivalent $\alpha$ values.

\begin{tabular}{lll}
\hline ID & Linguistic quantifier & Parameter \\
\hline 1 & All (max) & $\alpha \rightarrow \infty:(1000)$ \\
2 & Most & $\alpha=5$ \\
3 & Many & $\alpha=2$ \\
4 & Half (weighted averaging) & $\alpha=1$ \\
5 & Some & $\alpha=0.5$ \\
6 & Few & $\alpha=0.2$ \\
7 & At least one (min) & $\alpha \rightarrow 0:(0.001)$ \\
\hline
\end{tabular}

in which $\alpha$ is a parameter indicating the decision strategies (rules); its changes represent a continuum of different decision strategies between the two extreme cases of requiring "all" and "at least one" of the criteria to be satisfied. The common decision strategies and their corresponding parameter $\alpha$ are listed in Table $2[4,33]$.

Based on linguistic quantifier $Q$, the decision makers provide a decision strategy with a linguistic quantifier that indicates the portion of the criteria they feel is necessary for a good solution.

\section{Approach for Selecting Shale Gas Areas Based on the IVHFHSWA Operator}

The following assumptions or notations are used to represent the MCDM problems with interval-valued hesitant fuzzy information. Let $\left\{A_{1}, A_{2}, \ldots, A_{m}\right\}$ be a discrete set of alternatives and let $\left\{C_{1}, C_{2}, \ldots, C_{n}\right\}$ be the set of criteria and its relative weight vector is $\omega=\left(\omega_{1}, \omega_{2}, \ldots, \omega_{n}\right)$. Decision makers provide interval values for the alternative $A_{i}$ under the criteria $C_{j}$ with anonymity. To avoid performing information aggregation and to directly reflect the differences of the opinions of different experts, these interval values are considered as an interval-valued hesitant fuzzy element $\widetilde{h}_{i j}$ and formed the decision matrix $\left(\widetilde{h}_{i j}\right)_{m \times n}$.

In the following, we apply the IVHFHSWA (IVHFHSWG) operator to multiple criteria decision making based on interval-valued hesitant fuzzy information. The method involves the following steps.

Step 1. Determine the associated weights by utilizing (69) and (70) as

$$
w_{j}=\left(\frac{j}{n}\right)^{\alpha}-\left(\frac{j-1}{n}\right)^{\alpha}, \quad j=1, \ldots, n
$$

Step 2. Rank the criteria values against alternatives by the relative possibility degrees, (17), and then assign the associated weights as

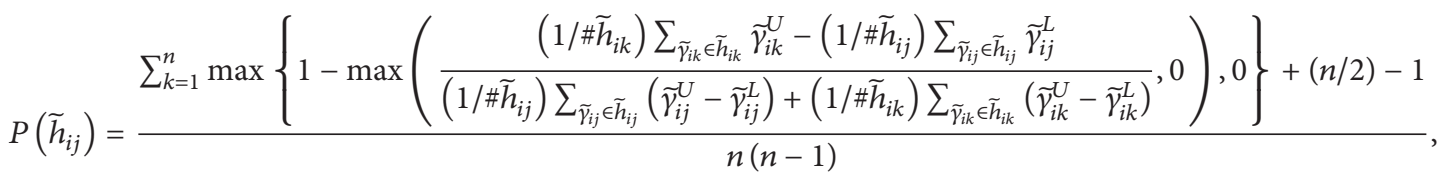

$$
\begin{aligned}
& i=1,2, \ldots, m, j=1,2, \ldots, n . \\
& \tilde{h}_{i}=\underset{\text { Hamacher }}{\operatorname{IVHFSWG}}\left(\widetilde{h}_{i 1}, \tilde{h}_{i 2}, \ldots, \widetilde{h}_{i n}\right)=\bigotimes_{j=1}^{n} \tilde{h}_{i j}^{\omega_{j} w_{\rho(j)} / \sum_{j=1}^{n} \omega_{j} w_{\rho(j)}} \text {, } \\
& i=1,2, \ldots, m
\end{aligned}
$$

Step 3. Utilize the IVHFHSWA operator, (34), as

$$
\begin{array}{r}
\widetilde{h}_{i}=\underset{\text { Hamacher }}{\operatorname{IVHFSWA}}\left(\widetilde{h}_{i 1}, \widetilde{h}_{i 2}, \ldots, \widetilde{h}_{i n}\right)=\left(\frac{\bigoplus_{j=1}^{n} \omega_{j} \tilde{h}_{i j} w_{\rho(j)}}{\sum_{j=1}^{n} \omega_{j} w_{\rho(j)}}\right), \\
i=1,2, \ldots, m .
\end{array}
$$

to aggregate decision information of $\widetilde{h}_{i j}(i=1,2, \ldots, m, j=$ $1,2, \ldots, n)$ and obtain the IVHFEs $\widetilde{h}_{i}(i=1,2 \ldots, m)$ for the alternatives $A_{i}(i=1,2, \ldots, m)$.

Or the IVHFHSWG operator, (51), as

Step 4. Compute the relative possibility degrees $P\left(\widetilde{h}_{i}\right)(i=$ $1,2, \ldots, m)$ of the IVHFEs $\widetilde{h}_{i}(i=1,2, \ldots, m)$ by utilizing $(17)$ as

$$
P\left(\widetilde{h}_{i}\right)=\frac{\sum_{k=1}^{m} \max \left\{1-\max \left(\frac{\left(1 / \# \tilde{h}_{k}\right) \sum_{\widetilde{\gamma}_{k} \in \tilde{h}_{k}} \widetilde{\gamma}_{k}^{U}-\left(1 / \# \widetilde{h}_{i}\right) \sum_{\widetilde{\gamma}_{i} \in \tilde{h}_{i}} \widetilde{\gamma}_{i}^{L}}{\left(1 / \# \widetilde{h}_{i}\right) \sum_{\widetilde{\gamma}_{i} \in \tilde{h}_{i}}\left(\widetilde{\gamma}_{i}^{U}-\widetilde{\gamma}_{i}^{L}\right)+\left(1 / \# \widetilde{h}_{k}\right) \sum_{\widetilde{\gamma}_{k} \in \widetilde{h}_{k}}\left(\widetilde{\gamma}_{k}^{U}-\widetilde{\gamma}_{k}^{L}\right)}, 0\right), 0\right\}+(m / 2)-1}{m(m-1)},
$$


TABLE 3: IVHF decision matrix.

\begin{tabular}{lcccc}
\hline & $C_{1}$ & $C_{2}$ & $C_{3}$ & $C_{4}$ \\
\hline$A_{1}$ & $\{[0.4,0.6],[0.8,0.9]\}$ & $\{[0.5,0.7]\}$ & $\{[0.6,0.8]\}$ & $\{[0.3,0.4],[0.4,0.5],[0.5,0.6]\}$ \\
$A_{2}$ & $\{[0.7,0.8]\}$ & $\{[0.4,0.6]\}$ & $\{[0.5,0.7],[0.8,0.9]\}$ & $\{[0.3,0.6],[0.7,0.9]\}$ \\
$A_{3}$ & $\{[0.3,0.4],[0.6,0.8]\}$ & $\{[0.3,0.4],[0.6,0.7]\}$ & $\{[0.6,0.8]\}$ & $\{[0.2,0.3],[0.5,0.7]\}$ \\
$A_{4}$ & $\{[0.4,0.5]\}$ & $\{[0.2,0.3],[0.4,0.5]\}$ & $\{[0.5,0.6],[0.7,0.8]\}$ & $\{[0.3,0.5]\}$ \\
$A_{5}$ & $\{[0.6,0.7],[0.8,0.9]\}$ & $\{[0.3,0.5]\}$ & $\{[0.7,0.8]\}$ & $\{[0.3,0.4],[0.5,0.7]\}$ \\
\hline
\end{tabular}

Step 5. Rank all the alternatives $A_{i}(i=1,2, \ldots, m)$ and select the best one(s) according to $P\left(\widetilde{h}_{i}\right)(i=1,2, \ldots, m)$ in descending order.

Step 6. End.

\section{Numerical Example}

With the increase of energy consumption, conventional natural gas resources are gradually reducing and getting increasingly difficult to develop. Due to the shale gas with the advantages of great resource potential and low carbon emissions, shale gas has recently been the focus of exploration in many countries and the development of shale gas becomes the strategic choice of China $[35,36]$. It is well known that the evaluation and selection of the areas are fundamental in the process of shale gas development. In order to preliminary select a best area from the five potential shale gas areas in South China: the Hunan area, the Sichuan area, the Yunnan area, the Guizhou area, and the Guangxi area, denoted as $x_{i}(i=1,2,3,4,5)$, respectively. The policy makers from government agencies, environmental organizations, local communities, and so forth serve as the decision makers. The evaluation criteria is based on the SWOT perspectives [37] and they are summarized as follows (adopted from [36]): strengths $\left(C_{1}\right)$, which consists of abundant resource reserves, great development potential, high environmental benefits, and long lifetime for exploitation; weaknesses $\left(C_{2}\right)$, which can be divided into lack of funds, lack of key technologies, prominent water treatment problems, and serious environmental risks; opportunities $\left(C_{3}\right)$, which involve huge potential market, policy support, increased investment and financing channels, plentiful foreign development experience, and deepened international cooperation; threats $\left(C_{4}\right)$, which can include unconfirmed resource potential, imperfect policy system, unsound management system, deficient investment mechanism, poor infrastructure, and the relative weight vector of the criteria which is $\omega=\left(\omega_{1}, \omega_{2}, \omega_{3}, \omega_{4}\right)=$ $(0.1,0.3,0.2,0.4)$. Given the experts who make such an evaluation have different backgrounds and levels of knowledge, skills, experience, personality, and so forth, this could lead to a difference in the evaluation information. To clearly reflect the differences of the opinions of different experts, the data of evaluation information are represented by the IVHFEs and listed in Table 3.

Below we apply the proposed approach to the selection of shale gas areas.
Step 1. Determine the associated weights by (71), where the term "many" is selected as a linguistic quantifier. The results are listed as follows:

$$
\begin{aligned}
& w_{1}=\left(\frac{1}{4}\right)^{2}-\left(\frac{0}{4}\right)^{2}=0.06 \\
& w_{2}=\left(\frac{2}{4}\right)^{2}-\left(\frac{1}{4}\right)^{2}=0.19 \\
& w_{3}=\left(\frac{3}{4}\right)^{2}-\left(\frac{2}{4}\right)^{2}=0.31 \\
& w_{4}=\left(\frac{4}{4}\right)^{2}-\left(\frac{3}{4}\right)^{2}=0.44 .
\end{aligned}
$$

Step 2. Rank the criteria values against each alternative by (72), and then assign the associated weights according to the rankings. The results are listed in Table 4.

Step 3. Utilize (73) and suppose that $\eta=1$ to aggregate decision information of $\widetilde{h}_{i j}(i=1,2,3,4,5, j=1,2,3,4)$ and obtain the IVHFEs $\widetilde{h}_{i}(i=1,2,3,4,5)$ for the alternatives $A_{i}(i=1,2,3,4,5)$.

Consider

$$
\begin{array}{r}
\widetilde{h}_{1}=\underset{\text { Hamacher }}{\operatorname{IVHFSWA}}\left(\widetilde{h}_{11}, \widetilde{h}_{12}, \widetilde{h}_{13}, \widetilde{h}_{14}\right) \\
=\{[0.385,0.540],[0.441,0.591], \\
\quad[0.499,0.643],[0.419,0.572], \\
\quad[0.473,0.620],[0.528,0.670]\}, \\
\widetilde{h}_{2}=\underset{\text { Hamacher }}{\operatorname{IVHFSWA}}\left(\widetilde{h}_{21}, \widetilde{h}_{22}, \widetilde{h}_{23}, \widetilde{h}_{24}\right) \\
=\{[0.382,0.619],[0.559,0.779], \\
\quad[0.441,0.665],[0.606,0.808]\}, \\
\widetilde{h}_{3}=\underset{\text { Hamacher }}{\operatorname{IVHFSWA}}\left(\widetilde{h}_{31}, \widetilde{h}_{32}, \widetilde{h}_{33}, \widetilde{h}_{34}\right) \\
=\{[0.256,0.366],[0.435,0.612], \\
\quad[0.363,0.478],[0.526,0.691], \\
\quad 0.277,0.400],[0.454,0.637],
\end{array}
$$$$
[0.383,0.509],[0.543,0.712]\} \text {, }
$$ 
TABLE 4: Rankings and the assigned associated weights of criteria values against alternatives.

\begin{tabular}{lcccc}
\hline & Rankings of criteria values against alternatives & $C_{1}$ & $C_{2}$ & $C_{3}$ \\
\hline$A_{1}$ & $\widetilde{h}_{13}>\widetilde{h}_{11}>\widetilde{h}_{12}>\widetilde{h}_{14}$ & 0.19 & 0.31 & 0.06 \\
$A_{2}$ & $\widetilde{h}_{21}>\widetilde{h}_{23}>\widetilde{h}_{24}>\widetilde{h}_{22}$ & 0.06 & 0.44 & 0.44 \\
$A_{3}$ & $\widetilde{h}_{33}>\widetilde{h}_{31}>\widetilde{h}_{32}>\widetilde{h}_{34}$ & 0.19 & 0.31 & 0.19 \\
$A_{4}$ & $\widetilde{h}_{43}>\widetilde{h}_{41}>\widetilde{h}_{44}>\widetilde{h}_{42}$ & 0.19 & 0.44 & 0.06 \\
$A_{5}$ & $\widetilde{h}_{51}=\widetilde{h}_{53}>\widetilde{h}_{54}>\widetilde{h}_{52}$ & 0.125 & 0.44 & 0.06 \\
\hline
\end{tabular}

TABLE 5: Score values obtained by the IVHFHSWA operator with different values of $\eta$.

\begin{tabular}{lccccc}
\hline & $A_{1}$ & $A_{2}$ & $A_{3}$ & $A_{4}$ & $A_{5}$ \\
\hline 1 & {$[0.4611,0.6115]$} & {$[0.5042,0.722]$} & {$[0.4108,0.5582]$} & {$[0.3254,0.4703]$} & {$[0.4156,0.5836]$} \\
2 & {$[0.4575,0.6060]$} & {$[0.4968,0.7181]$} & {$[0.4048,0.5506]$} & {$[0.3224,0.4673]$} & {$[0.4074,0.5776]$} \\
3 & {$[0.4558,0.6035]$} & {$[0.4932,0.7164]$} & {$[0.4014,0.5469]$} & {$[0.3207,0.4657]$} & {$[0.4033,0.5749]$} \\
4 & {$[0.4548,0.6021]$} & {$[0.4911,0.7155]$} & {$[0.3993,0.5447]$} & {$[0.3197,0.4648]$} & {$[0.4009,0.5734]$} \\
5 & {$[0.4541,0.6012]$} & {$[0.4897,0.7149]$} & {$[0.3978,0.5432]$} & {$[0.3190,0.4641]$} & {$[0.3992,0.5725]$} \\
6 & {$[0.4536,0.6005]$} & {$[0.4887,0.7145]$} & {$[0.3966,0.5421]$} & {$[0.3184,0.4637]$} & {$[0.3981,0.5718]$} \\
7 & {$[0.4532,0.6000]$} & {$[0.4879,0.7142]$} & {$[0.3958,0.5413]$} & {$[0.3180,0.4633]$} & {$[0.3972,0.5713]$} \\
8 & {$[0.4529,0.5997]$} & {$[0.4874,0.7140]$} & {$[0.3951,0.5407]$} & {$[0.3177,0.4630]$} & {$[0.3965,0.5709]$} \\
9 & {$[0.4526,0.5994]$} & {$[0.4869,0.7139]$} & {$[0.3945,0.5402]$} & {$[0.3174,0.4628]$} & {$[0.3959,0.5706]$} \\
10 & {$[0.4525,0.5991]$} & {$[0.4865,0.7137]$} & {$[0.3941,0.5398]$} & {$[0.3172,0.4626]$} & {$[0.3955,0.5704]$} \\
\hline
\end{tabular}

$$
\begin{array}{r}
\tilde{h}_{4}=\underset{\text { Hamacher }}{\operatorname{IVHFSWA}}\left(\widetilde{h}_{41}, \widetilde{h}_{42}, \widetilde{h}_{43}, \widetilde{h}_{44}\right) \\
=\{[0.271,0.418],[0.283,0.431], \\
\quad[0.362,0.504],[0.373,0.516]\}, \\
\tilde{h}_{5}=\underset{\text { Hamacher }}{\operatorname{IVHFSWA}}\left(\widetilde{h}_{51}, \widetilde{h}_{52}, \widetilde{h}_{53}, \widetilde{h}_{54}\right) \\
=\{[0.358,0.507],[0.443,0.632], \\
\quad[0.372,0.525],[0.456,0.646]\} .
\end{array}
$$

Step 4. Compute the relative possibility degrees $P\left(\widetilde{h}_{i}\right)(i=$ $1,2, \ldots, 5)$ of the IVHFEs $\widetilde{h}_{i}(i=1,2, \ldots, 5)$.

$$
\begin{gathered}
P\left(\widetilde{h}_{1}\right)=0.229, \quad P\left(\widetilde{h}_{2}\right)=0.267, \quad P\left(\widetilde{h}_{3}\right)=0.185, \\
P\left(\widetilde{h}_{4}\right)=0.122, \quad P\left(\widetilde{h}_{5}\right)=0.197
\end{gathered}
$$

Step 5. Rank all the alternatives $A_{i}(i=1,2, \ldots, m)$ according to $P\left(\tilde{h}_{i}\right)(i=1,2, \ldots, m)$ in descending order. The ranking results are $A_{2}>A_{1}>A_{5}>A_{3}>A_{4}$, and the most prospective shale gas area is $A_{2}$ (Sichuan).

Step 6. End.
Furthermore, we use the IVHFHSWG operator to the same decision problem, we can obtain the ranking results that consist with the ones above, which are validate each other.

\section{Comparative Analysis}

6.1. Performance Analysis of the IVHFHSWA Operator. From the definition of IVHFHSWA operator, we know that the IVHFHSWA operator provides a wide class of interval-valued hesitant fuzzy aggregation operators via the parameters $\eta$. To understand the performance of aggregation in depth, we adopt the parameter $\eta=1$ to 10 for the numerical example above. When the $\eta$ takes the different values, the scores values are obtained based on IVHFHSWA operator as shown in Table 5 and represented graphically in Figure 1.

From Table 5, it is obvious that the score values derived by using the IVHFHSWA operator are nonincreasing with respect to $\eta$, which implies that decision makers can utilize their preferences to give the preferred values of $\eta$ according to practical decision situations. On the basis of the score values, we can obtain the precisely ranking results of the alternatives by computing their relative possibility degrees. Moreover, from Figure 1, we find that the ranking results of the alternatives are the same when the values of $\eta$ are different in the example, and the consistent ranking results demonstrate the stability of the proposed operators.

6.2. Comparison With Other Operators. Similar to the IVHFHSWA operator, in order to integrate simultaneously 
TABLE 6: Score values obtained by the IVHFHSWA, IVHFHWA, and IVHFHOWA operator.

\begin{tabular}{lccccc}
\hline & $A_{1}$ & $A_{2}$ & $A_{3}$ & $A_{4}$ & $A_{5}$ \\
\hline IVHFSWA & {$[0.4611,0.6115]$} & {$[0.5042,0.7222]$} & {$[0.4108,0.5582]$} & {$[0.3254,0.4703]$} & {$[0.4156,0.5836]$} \\
IVHFWA & {$[0.5045,0.6744]$} & {$[0.5496,0.7500]$} & {$[0.4582,0.6232]$} & {$[0.3882,0.5290]$} & {$[0.4940,0.6455]$} \\
IVHFOWA & {$[0.4999,0.6588]$} & {$[0.5254,0.7284]$} & {$[0.4312,0.5847]$} & {$[0.3455,0.4781]$} & {$[0.4651,0.6258]$} \\
\hline
\end{tabular}

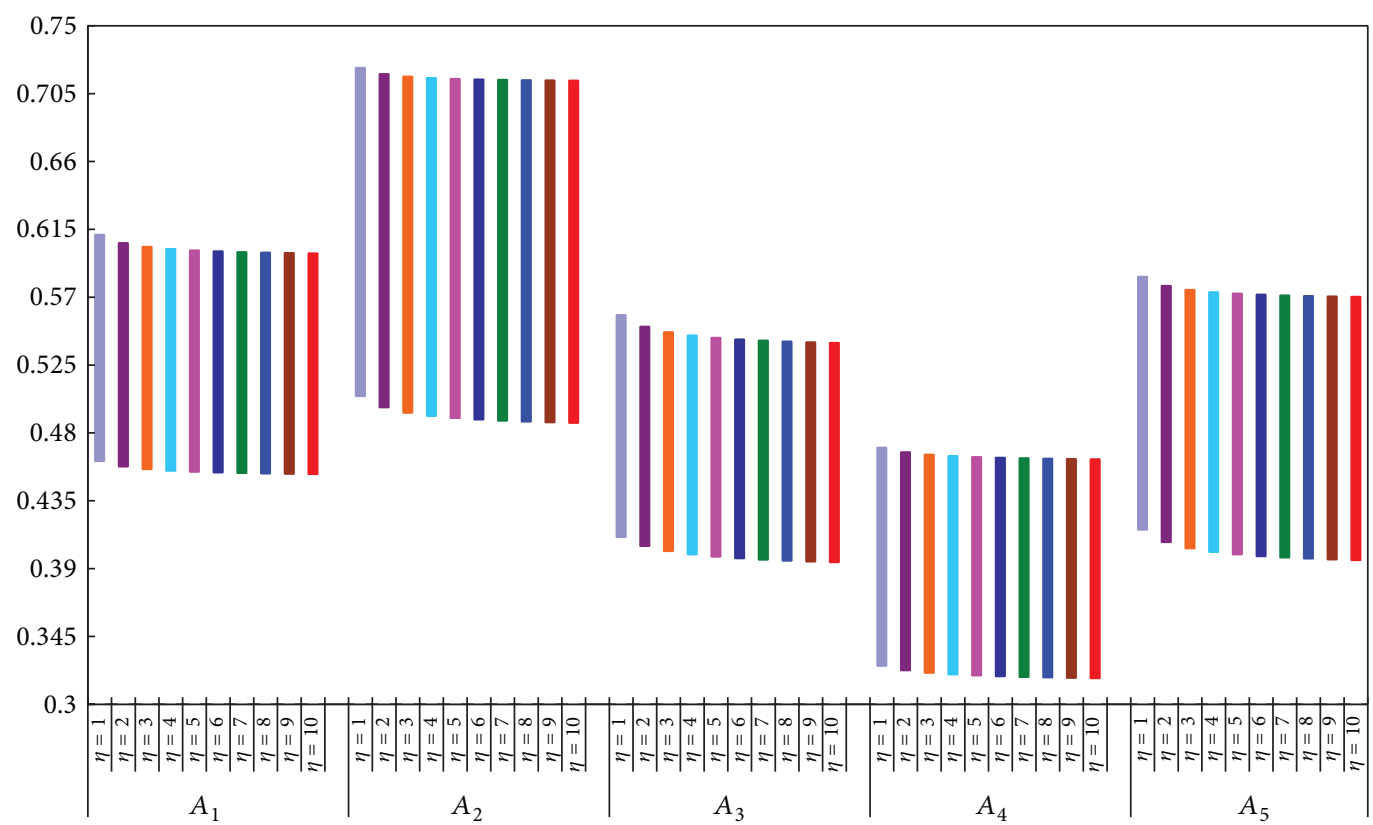

FIGURE 1: Score values obtained by the IVHFHSWA operator with different values of $\eta$.

the relative weights and the associated weights into averaging operator, Chen et al. [11] have developed an interval-valued hesitant fuzzy hybrid averaging (IVHFHA) operator based on the hybrid weighted aggregation [37] operator, which is defined as follows:

$$
\begin{aligned}
& \operatorname{IVHFHA}\left(\widetilde{h}_{1}, \widetilde{h}_{2}, \ldots, \widetilde{h}_{n}\right) \\
& =\bigoplus_{j=1}^{n} w_{j} \dot{\tilde{h}}_{\sigma(j)} \\
& =\underset{\dot{\tilde{\gamma}}_{\sigma(j)} \in \dot{\tilde{h}}_{\sigma(j)}, j=1, \ldots, n}{\bigcup}\left\{\left[1-\prod_{j=1}^{n}\left(1-\dot{\tilde{\gamma}}_{\sigma(j)}^{L}\right)^{w_{j}},\right.\right. \\
& \left.\left.1-\prod_{j=1}^{n}\left(1-\dot{\tilde{\gamma}}_{\sigma(j)}^{U}\right)^{w_{j}}\right]\right\},
\end{aligned}
$$

where $\dot{\widetilde{h}}_{\sigma(j)}$ is the $j$ th largest of $\dot{\widetilde{h}}_{j}=n \omega_{j} \widetilde{h}_{j}(j=1,2, \ldots, n)$. However, the HWA operator has proven that it does not satisfy boundary, idempotent, and so forth $[4,38]$. Moreover, the computation of IVHFHWA operator is simpler than the ones of IVHFHA operator. When using the IVHFHA operator, we have to first calculate $\dot{\widetilde{h}}_{j}=n \omega_{j} \widetilde{h}_{j}$ and compare them, and then calculate $w_{j} \dot{\widetilde{h}}_{\sigma(j)}$, after which, we will compute the aggregation values with $\oplus_{j=1}^{n} w_{j} \dot{\widetilde{h}}_{\sigma(j)}$. Since the computation with interval-valued hesitant fuzzy sets is very complex, the results derived via the IVHFHA operator are hard to be obtained. As for the IVHFHSWA operator, the operation of the relative weights and the ones of associated weights in IVHFHWA operator are synchronized, which is in the mathematical form as $\omega_{j} w_{\rho(j)}$. Since both $\omega_{j}$ and $w_{\rho(j)}$ are crisp numbers, we only need to calculate $\left(\oplus_{j=1}^{n} \omega_{j} \widetilde{h}_{j} w_{\rho(j)}\right) /\left(\sum_{j=1}^{n} \omega_{j} w_{\rho(j)}\right)$, which makes the IVHFHSWA operator easier to calculate than the IVHFHA operator. Furthermore, the IVHFHWA operator can provide more special cases by selecting different values of parameter $\eta$, which can provide more choices for the decision makers and considerably enhance or deteriorate the performance of aggregation and thus is more general and more flexible.

To show the advantage of IVHFHSWA operator against the VHFHWA and IVHFHOWA operators, we use again the IVHFHSWA, IVHFHWA, and IVHFHOWA operators to the numerical example above; here we take $\eta=1$ as example, and their final scores are listed in Table 6 and represented graphically in Figure 2.

From Table 6 and Figure 2, it is clear that despite the score values obtained by the IVHFHSWA, IVHFHWA, and IVHFHOWA operators are different; it is clear that despite the score values of the alternatives obtained by the IVHFHSWA, IVHFHWA, and IVHFHOWA operators are different; 


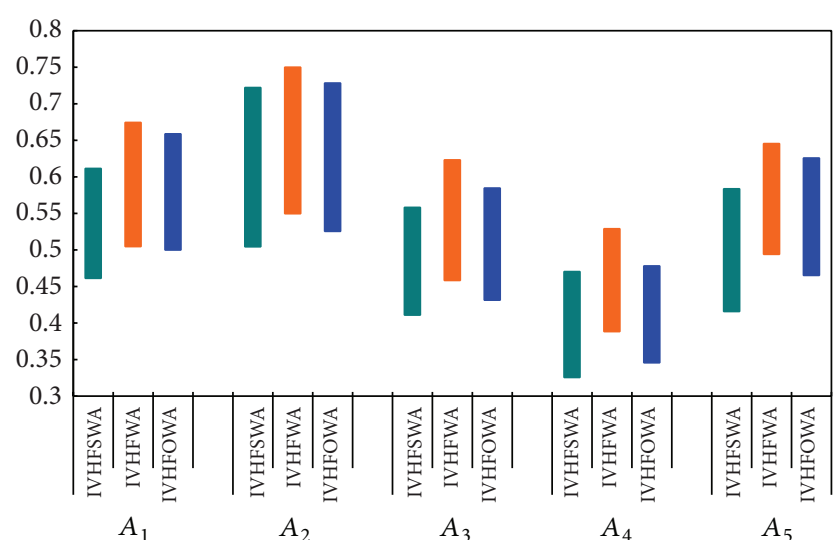

FIGURE 2: Score values obtained by the IVHFHSWA, IVHFHWA, and IVHFHOWA operator.

the ranking results of the alternatives derived from them are the same; that is, $A_{2}>A_{1}>A_{5}>A_{3}>A_{4}$. The reasons about the difference of score values is intuitive that, as discussed above, the IVHFHWA operator focuses solely on the relative weights and ignores the associated weights, while the IVHFHOWA operator focuses only on the associated weights and ignores the relative weights. The IVHFHSWA operator comprehensively considers both the associated weights and the relative weights. Hence, the results derived by IVHFHSWA operator are more feasible and effective. On the other hand, the identical ranking results imply that the IVHFHSWA, IVHFHWA, and IVHFHOWA operators all are effective.

Finally, our interval-valued hesitant fuzzy aggregation (IVHFHWA, IVHFHOWA, IVHFHSWA, IVHFHWG, IVHFHOWG, and IVHFHSWG) operators can also be applied to deal with the situations when the interval-valued hesitant fuzzy sets are reduced to hesitant fuzzy sets. In contrast, the existing hesitant fuzzy aggregation operators (HFWA, HFOWA, HFHA, HFWG, HFOWG, and HFHG) cannot be applied to deal with the interval-valued hesitant fuzzy situation. In other words, our interval-valued hesitant fuzzy aggregation operators have much wider applications than the existing hesitant fuzzy aggregation operators.

\section{Conclusion}

In this paper, we first introduce the Hamacher operations of interval-valued hesitant fuzzy sets and developed some interval-valued hesitant fuzzy Hamacher operators, including the interval-valued hesitant fuzzy Hamacher weighted averaging (IVHFHWA) operator and interval-valued hesitant fuzzy Hamacher ordered weighted averaging (IVHFHOWA) operator. The prominent advantages of the developed operators are that they provide a family of interval-valued hesitant fuzzy aggregation operators that include the existing intervalvalued hesitant fuzzy operators and interval-valued hesitant fuzzy Einstein operators as special cases and then provide more choices for the decision makers. Then, we proposed an interval-valued hesitant fuzzy Hamacher synergetic weighted averaging operator to generalize further the IVHFHWA and IVHFHOWA operator. Some essential properties of the proposed operators are studied and their special cases are discussed. Based on the IVHFHSWA operator, we develop a practical approach to multiple criteria decision making with interval-valued hesitant fuzzy information. Finally, an illustrative example for selecting the shale gas areas is used to illustrate the proposed approach and a comparative analysis is performed with other approaches to highlight the distinctive advantages of the proposed operators.

\section{Conflict of Interests}

The authors declare that there is no conflict of interests regarding the publication of this paper.

\section{Acknowledgments}

The authors are very grateful to the editor Wudhichai Assawinchaichote and the anonymous referees for their insightful and constructive comments and suggestions which have helped to improve the paper. This work was supported in part by the National Natural Science Funds of China (nos. 61364016, 71272191, and 71072085), the scientific Research Fund Project of Educational Commission of Yunnan Province, China (no. 2013Y336), the Science and Technology Planning Project of Yunnan Province, China (no. 2013SY12), the Natural Science Funds of KUST (no. KKSY201358032), and the Graduate Innovation Funds of Heilongjiang Province of China (no. YJSCX2011-003HLJ).

\section{References}

[1] V. Torra and Y. Narukawa, "On hesitant fuzzy sets and decision," in Proceedings of the 18th IEEE International Conference on Fuzzy Systems, pp. 1378-1382, Jeju Island, Republic of Korea, August 2009.

[2] V. Torra, "Hesitant fuzzy sets," International Journal of Intelligent Systems, vol. 25, no. 6, pp. 529-539, 2010.

[3] Z. S. Xu and M. Xia, "On distance and correlation measures of hesitant fuzzy information," International Journal of Intelligent Systems, vol. 26, no. 5, pp. 410-425, 2011.

[4] D.-H. Peng, C.-Y. Gao, and Z.-F. Gao, "Generalized hesitant fuzzy synergetic weighted distance measures and their application to multiple criteria decision-making," Applied Mathematical Modelling, vol. 37, no. 8, pp. 5837-5850, 2013.

[5] X. Zhang and Z. S. Xu, "Hesitant fuzzy agglomerative hierarchical clustering algorithms," International Journal of Systems Science, 2013.

[6] M. Xia and Z. S. Xu, "Hesitant fuzzy information aggregation in decision making," International Journal of Approximate Reasoning, vol. 52, no. 3, pp. 395-407, 2011.

[7] Z. S. Xu and M. Xia, "Distance and similarity measures for hesitant fuzzy sets," Information Sciences, vol. 181, no. 11, pp. 2128-2138, 2011.

[8] D. Yu, W. Zhang, and Y. Xu, "Group decision making under hesitant fuzzy environment with application to personnel evaluation," Knowledge-Based Systems, vol. 52, pp. 1-10, 2013.

[9] B. Farhadinia, "A novel method of ranking hesitant fuzzy values for multiple attribute decision-making problems," International Journal of Intelligent Systems, vol. 28, no. 8, pp. 752-767, 2013. 
[10] G. Qian, H. Wang, and X. Feng, "Generalized hesitant fuzzy sets and their application in decision support system," KnowledgeBased Systems, vol. 37, pp. 357-365, 2013.

[11] N. Chen, Z. S. Xu, and M. Xia, "Interval-valued hesitant preference relations and their applications to group decision making," Knowledge-Based Systems, vol. 37, pp. 528-540, 2013.

[12] M. Xia, Z. S. Xu, and N. Chen, "Some hesitant fuzzy aggregation operators with their application in group decision making," Group Decision and Negotiation, vol. 22, no. 2, pp. 259-279, 2013.

[13] G. Wei, "Hesitant fuzzy prioritized operators and their application to multiple attribute decision making," Knowledge-Based Systems, vol. 31, pp. 176-182, 2012.

[14] B. Zhu, Z. S. Xu, and M. Xia, "Hesitant fuzzy geometric Bonferroni means," Information Sciences, vol. 205, pp. 72-85, 2012.

[15] Z. Zhang, "Hesitant fuzzy power aggregation operators and their application to multiple attribute group decision making," Information Sciences, vol. 234, pp. 150-181, 2013.

[16] B. Farhadinia, "Information measures for hesitant fuzzy sets and interval-valued hesitant fuzzy sets," Information Sciences, vol. 240, pp. 129-144, 2013.

[17] N. Chen, Z. S. Xu, and M. Xia, "Correlation coefficients of hesitant fuzzy sets and their applications to clustering analysis," Applied Mathematical Modelling, vol. 37, no. 4, pp. 2197-2211, 2013.

[18] Z. S. Xu and M. Xia, "Hesitant fuzzy entropy and cross-entropy and their use in multiattribute decision-making," International Journal of Intelligent Systems, vol. 27, no. 9, pp. 799-822, 2012.

[19] B. Zhu, Z. S. Xu, and M. Xia, "Dual hesitant fuzzy sets," Journal of Applied Mathematics, vol. 2012, Article ID 879629, 13 pages, 2012.

[20] R. M. Rodriguez, L. Martinez, and F. Herrera, "Hesitant fuzzy linguistic term sets for decision making," IEEE Transactions on Fuzzy Systems, vol. 20, no. 1, pp. 109-119, 2012.

[21] R. M. Rodríguez, L. Martínez, and F. Herrera, "A group decision making model dealing with comparative linguistic expressions based on hesitant fuzzy linguistic term sets," Information Sciences, vol. 241, pp. 28-42, 2013.

[22] G. W. Wei, “Some hesitant interval-valued fuzzy aggregation operators and their applications to multiple attribute decision making," Knowledge-Based Systems, vol. 46, pp. 43-53, 2013.

[23] G. W. Wei and X. Zhao, "Induced hesitant interval-valued fuzzy Einstein aggregation operators and their application to multiple attribute decision making," Journal of Intelligent \& Fuzzy Systems, vol. 24, no. 4, pp. 789-803, 2013.

[24] H. Hamachar, "Uber logische verknunpfungenn unssharfer aussagen und deren zugenhorige bewertungsfunktione," in Progress in Cybernatics and Systems Research, R. Trappl, G. J. Klir, and L. Riccardi, Eds., vol. 3, pp. 276-288, Hemisphere, Washington, DC, USA, 1978.

[25] J. Dombi, "Towards a general class of operators for fuzzy systems," IEEE Transactions on Fuzzy Systems, vol. 16, no. 2, pp. 477-484, 2008.

[26] E. P. Klement, R. Mesiar, and E. Pap, Triangular Norms, Kluwer Academic, Dodrecht, The Netherlands, 2000.

[27] T. Calvo, G. Mayor, and R. Mesiar, Aggregation Operators: New Trends and Applications, Physica, Heidelberg, Germany, 2002.

[28] R. R. Yager, "On ordered weighted averaging aggregation operators in multicriteria decision-making," IEEE Transactions on Systems, Man, and Cybernetics, vol. 18, no. 1, pp. 183-190, 1988.
[29] R. R. Yager, “Time series smoothing and OWA aggregation," IEEE Transactions on Fuzzy Systems, vol. 16, no. 4, pp. 994-1007, 2008.

[30] R. A. Ribeiro and R. A. Marques Pereira, "Generalized mixture operators using weighting functions: a comparative study with WA and OWA," European Journal of Operational Research, vol. 145, no. 2, pp. 329-342, 2003.

[31] Z. S. Xu, "An overview of methods for determining OWA weights," International Journal of Intelligent Systems, vol. 20, no. 8, pp. 843-865, 2005.

[32] R. R. Yager, "Quantifier guided aggregation using OWA operators," International Journal of Intelligent Systems, vol. 11, no. 1, pp. 49-73, 1996.

[33] D.-H. Peng, C.-Y. Gao, and L.-L. Zhai, "Multi-criteria group decision making with heterogeneous information based on ideal points concept," International Journal of Computational Intelligence Systems, vol. 6, no. 4, pp. 616-625, 2013.

[34] D.-H. Peng, Z.-F. Gao, C.-Y. Gao, and H. Wang, "A direct approach based on $\mathrm{C}^{2}$-IULOWA operator for group decision making with uncertain additive linguistic preference relations," Journal of Applied Mathematics, vol. 2013, Article ID 420326, 14 pages, 2013.

[35] A. Kalantari-Dahaghi and S. D. Mohaghegh, "A new practical approach in modelling and simulation of shale gas reservoirs: application to New Albany Shale," International Journal of Oil, Gas and Coal Technology, vol. 4, no. 2, pp. 104-133, 2011.

[36] X. Zhao, J. Kang, and B. Lan, "Focus on the development of shale gas in China-based on SWOT analysis," Renewable and Sustainable Energy Reviews, vol. 21, pp. 603-613, 2013.

[37] C.-Y. Gao and D.-H. Peng, "Consolidating SWOT analysis with nonhomogeneous uncertain preference information," Knowledge-Based Systems, vol. 24, no. 6, pp. 796-808, 2011.

[38] J. Lin and Y. Jiang, "Some hybrid weighted averaging operators and their application to decision making," Information Fusion, vol. 16, pp. 18-28, 2014. 


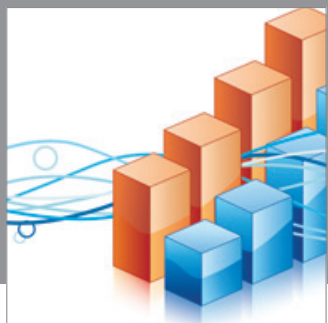

Advances in

Operations Research

mansans

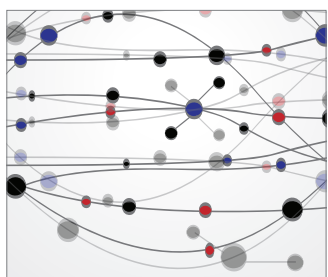

The Scientific World Journal
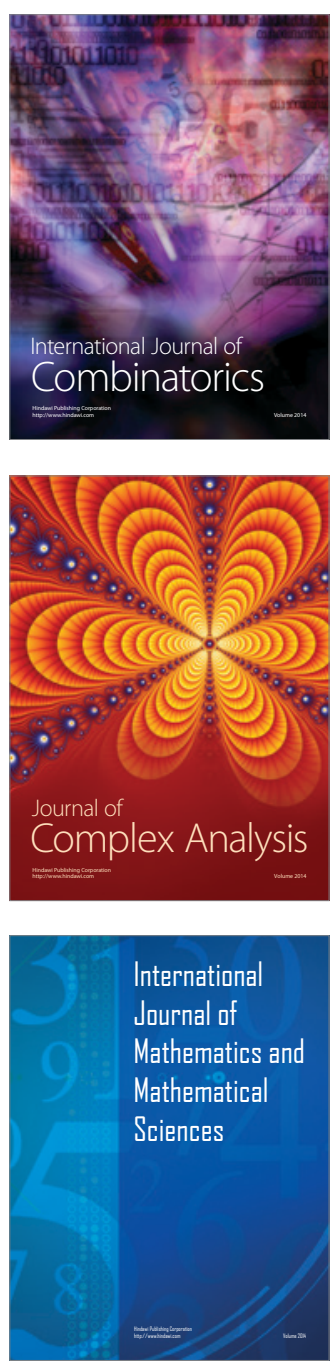
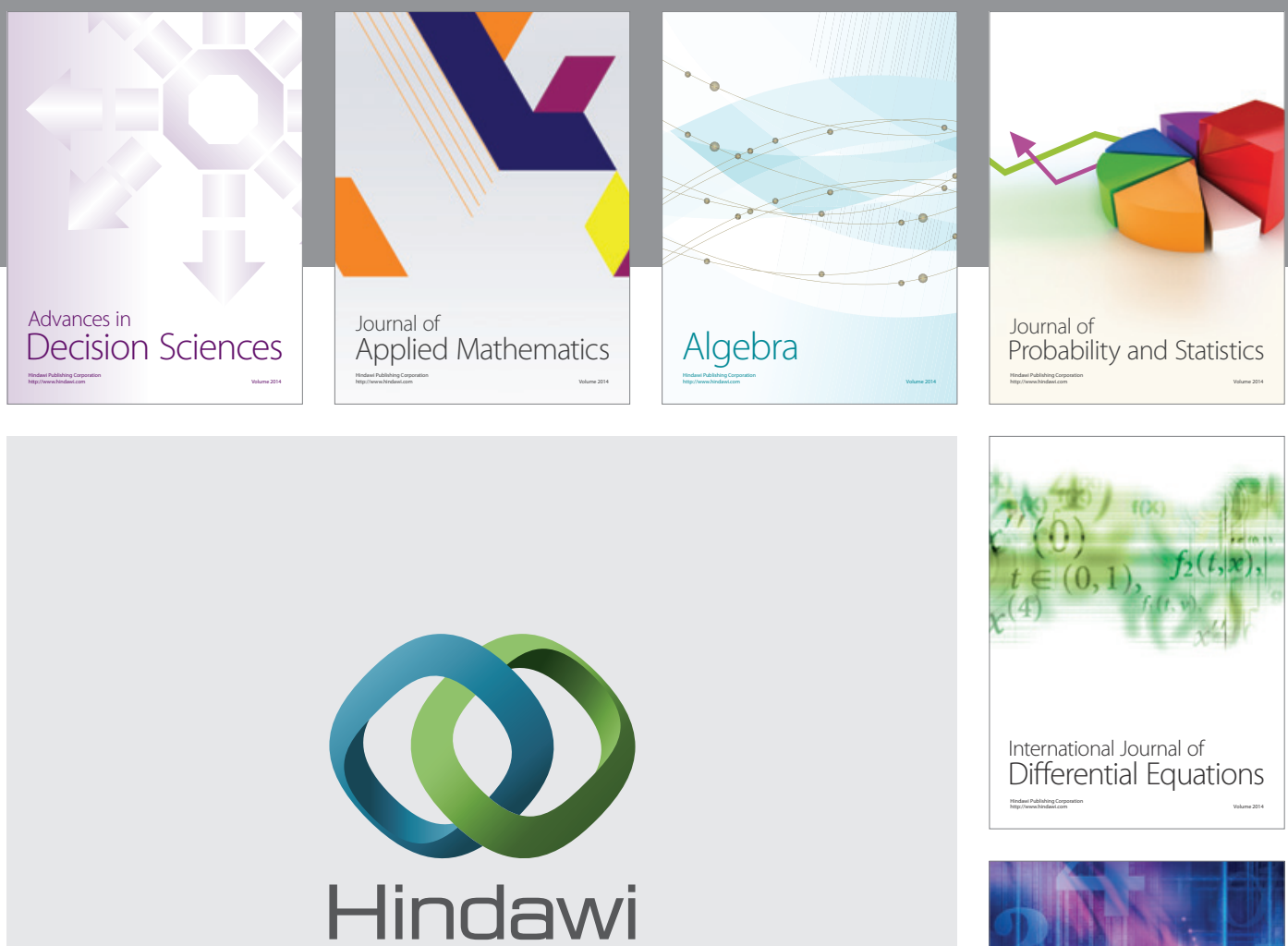

Submit your manuscripts at http://www.hindawi.com
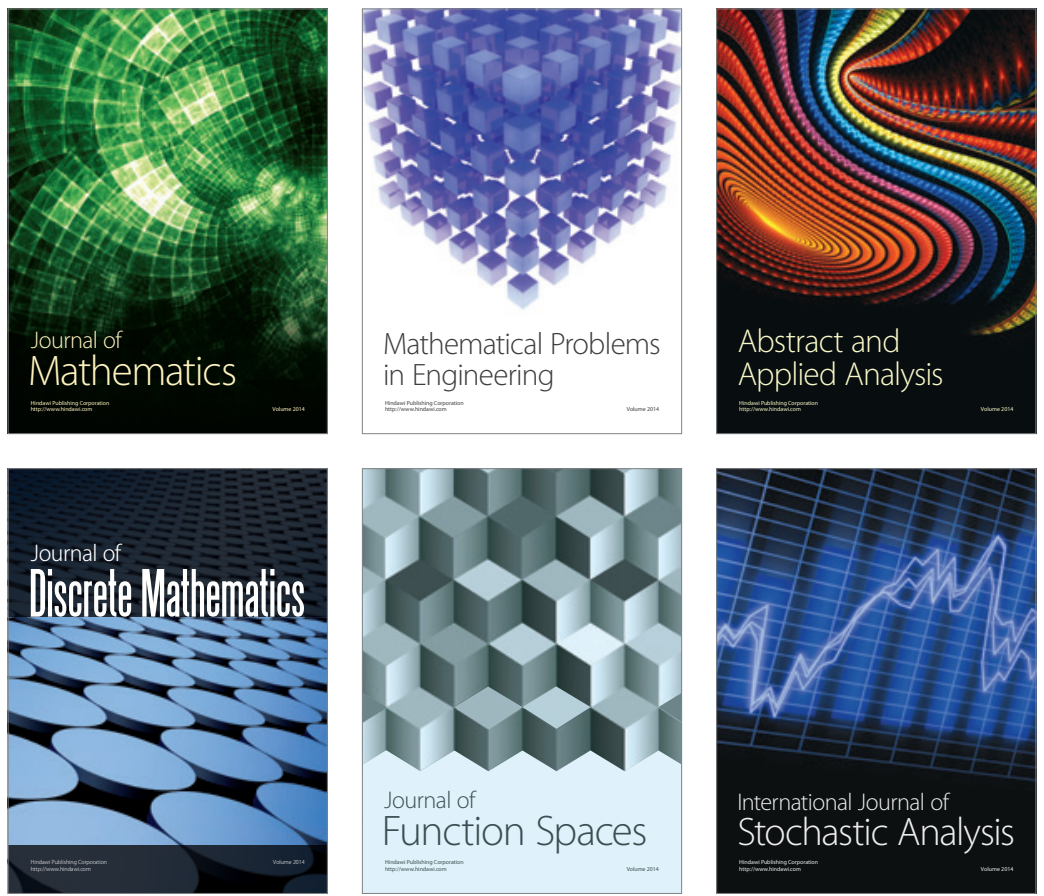

Journal of

Function Spaces

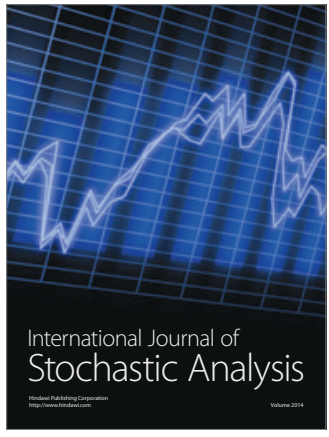

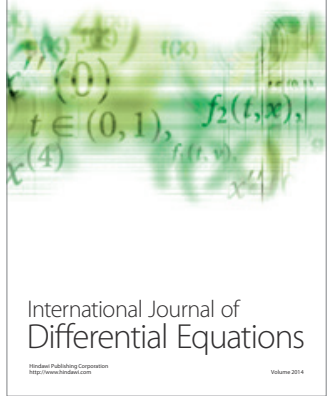
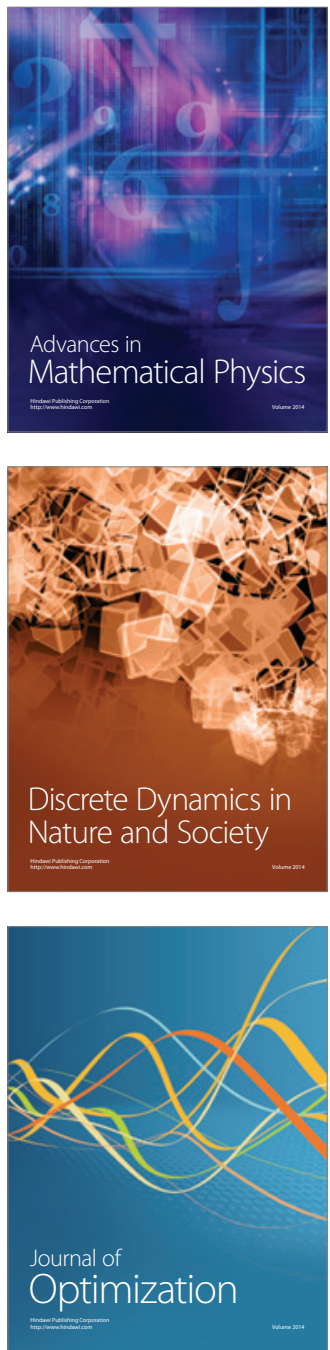